Article

\title{
Emerging Distortion Product Otoacoustic Emission Techniques to Identify Preclinical Warning Signs of Basal Cochlear Dysfunction Due to Ototoxicity
}

\author{
Gayla L. Poling ${ }^{1, *(D)}$, Brittany Vlosich ${ }^{2}$ and Laura E. Dreisbach ${ }^{2}$ \\ 1 Division of Audiology, Department of Otorhinolaryngology, Mayo Clinic, Rochester, MN 55905, USA \\ 2 School of Speech, Language, and Hearing Sciences, San Diego State University, San Diego, CA 92182, USA \\ * Correspondence: poling.gayla@mayo.edu
}

Received: 5 June 2019; Accepted: 31 July 2019; Published: 2 August 2019

\begin{abstract}
Hundreds of medications commonly prescribed for anticancer treatments and some infections are known to cause hearing damage, referred to as ototoxicity. Preventing or minimizing ototoxicity is critical in order to preserve quality of life for patients receiving treatment and to reduce the societal burden of hearing loss. Current clinical evaluations are restricted to a limited frequency range ( $\leq 8 \mathrm{kHz})$; however, this approach does not permit the earliest detection of ototoxicity, most likely to be observed at the highest frequencies $(9-20 \mathrm{kHz})$. Distortion product otoacoustic emissions (DPOAEs) offer a noninvasive, objective approach to monitor cochlear health in those unable to respond via conventional methods. The current report analyzes different DPOAE paradigms used in patients undergoing chemotherapy treatments with various platinum derivatives. Individualized serial monitoring protocols were completed at the highest frequencies with measurable DPOAEs. This allowed the exploration of potential clinical translation opportunities for further quantification of the earliest signs of underlying cochlear damage, which may go undetected with conventional methods. Clinical practice has the potential to be enhanced by emerging DPOAE applications, including targeted monitoring protocols and high-frequency stimuli to assess cochlear function, especially at the highest frequencies, and advanced calibration techniques to ensure the stability of serial measurements.
\end{abstract}

Keywords: distortion product otoacoustic emissions; serial monitoring; hearing loss; normal hearing; ototoxicity; cisplatin; carboplatin; oxaliplatin

\section{Introduction}

Hundreds of medications commonly prescribed for anticancer treatments and some infections are known to cause ototoxicity or damage to mechanisms of the auditory system. In fact, estimates report there are 200 to 600 potentially ototoxic medications (see overview by [1]). Among these ototoxic therapies are the commonly employed platinum derivatives, such as cisplatin, oxaliplatin, and carboplatin, often initially impacting outer hair cells (OHCs) at the base of the cochlea. This basal cochlear damage manifests as high-frequency hearing loss, which can be mitigated and/or management solutions adopted to optimize quality of life for patients if detected in a timely manner. Emerging research in the realm of otoprotectants and otorescue agents are further highlighting the need to establish methods for characterizing and monitoring cochlear health (e.g., [2]). Moreover, distortion product otoacoustic emissions (DPOAEs) offer a noninvasive, objective approach to monitor cochlear health utilizing frequencies and paradigms beyond those traditionally tested in patients unable to respond via conventional methods. 


\subsection{Serial Monitoring for Ototoxic Therapies}

Serial monitoring of auditory function provides opportunities for identification of cochlear dysfunction at the earliest indications of ototoxicity. Ideally, this includes a baseline evaluation prior to exposure to potentially ototoxic therapies followed by regular monitoring evaluations at an interval dictated by the treatment regimen (typically, from weeks to months) to compare to the baseline evaluation (refer to [3-5]). Current clinical evaluations are focused to a limited frequency range (up to $8 \mathrm{kHz}$ ); however, this approach does not permit the earliest detection of ototoxicity, most likely to be observed at the highest frequencies (up to $20 \mathrm{kHz}$ ). Of note, higher frequency audiometry allows for earlier detection of damage from ototoxic agents than conventional audiometry [6]. However, pure-tone behavioral thresholds are subjective and can be dependent on aspects of the patient other than their auditory function. Patient populations receiving chemotherapeutic treatments may be difficult to test with pure-tone audiometry, exhibiting "poor cooperation and easy distractibility" due to their health status and levels of fatigue, causing unreliable results [7]. In a Veteran population, a substantial number (approximately 33\%) of patients became unable or unwilling to participate in behavioral hearing testing [8]. This makes DPOAEs, an objective test not requiring patient participation, an ideal measure for monitoring.

Many therapeutic agents used to treat cancer are often ototoxic, among these are platinum derivatives, such as cisplatin, oxaliplatin, and carboplatin, frequently impacting $\mathrm{OHCs}$ at the base of the cochlea first $[3,5,9]$. The damage caused by cisplatin is generally focused on the OHCs within the cochlea, starting with the OHCs in the cochlear base, and spreading more apically as the dose increases [10]. Damage spreads beyond OHCs to surrounding structures, including the stria vascularis, with increased exposure (see review by $[3,9]$ ). Oxaliplatin has been shown in animal models to have comparable effects on the OHCs in the basal end of the cochlea [11]. The damage associated with carboplatin has been thought to impact inner hair cells (IHCs) more than OHCs in animal models [12,13]; however, carboplatin has been shown to cause OHC loss in chinchillas [14], especially at higher doses [12,15], and in humans [16]. In conjunction with these lifesaving treatment options, monitoring hearing is important to make adjustments to treatment dosage/protocol (if possible) and/or to provide intervention if their hearing declines [3]. Close monitoring of the auditory system while on an ototoxic drug regimen permits care teams the opportunity to manage treatment side effects, intervene when appropriate during the course of treatment as well as provide the patient with intervention methods (i.e., hearing aids, communication strategies, and aural rehabilitation) in a timely manner to optimize quality of life outcomes. DPOAEs are particularly important for ototoxic monitoring because they are correlated with OHC damage in animals (e.g., [17]), the portion of the auditory system predominately impacted by ototoxic therapies $[10,11]$. Monitoring $\mathrm{OHC}$ function directly can allow for the earliest actions to be taken in either preventing further damage or providing audiologic intervention. Due to the ability of DPOAE measures to assess the status of OHCs, they are distinctively qualified to give information about the health of $\mathrm{OHCs}$ and give insight into potential damage from ototoxic therapies.

\subsection{Expanding the Bandwidth Beyond $8 \mathrm{kHz}$}

Ototoxic monitoring for patients in the clinic has traditionally been completed at conventional frequency regions $(0.25-8 \mathrm{kHz})$ with higher frequencies $(>8 \mathrm{kHz})$ explored when hardware and time permits. This expanded frequency bandwidth permits broader characterization of cochlear health which is particularly valuable for ototoxicity, which often affects the base of the cochlea first (corresponding to higher frequencies of human hearing). Consequently, high-frequency audiometry permits for earlier detection of damage from ototoxic agents than conventional audiometry [6]. In this paper, we leverage modern techniques of data recording, calibration, and analysis to characterize DPOAE characteristics up to $20 \mathrm{kHz}$ utilizing patient case illustrations to demonstrate applications for high-frequency DPOAE assessment. The advancement of calibration techniques enabling accurate 
signal delivery at all frequencies (e.g., [18-22]) allow the extension of these measures to the upper limit of human hearing.

\subsection{Advancing Distortion Product Otoacoustic Emission (DPOAE) Paradigms}

Distortion product otoacoustic emissions (DPOAEs) offer a noninvasive, objective approach to monitor cochlear health. DPOAEs can be recorded in the ear canal in response to two simultaneous pure tones $\left(\mathrm{f}_{1}\right.$ and $\left.\mathrm{f}_{2}, \mathrm{f}_{1}<\mathrm{f}_{2}\right)$ at frequencies mathematically related to the stimulus frequencies [23]. The response that is the largest level in humans and most commonly used in the clinic for detection of hearing loss is the $2 \mathrm{f}_{1}-\mathrm{f}_{2}$ DPOAE [24]. DPOAEs in clinical practice provide a frequency-specific snapshot of overall outer hair cell function with measurements at a few stimulus frequency pairs per octave and using standard stimulus parameters (level of $f_{1} / f_{2}$ of $65 / 55 \mathrm{~dB} \mathrm{SPL}$, stimulus ratio $\left(\mathrm{f}_{2} / \mathrm{f}_{1}\right)$ of $1.22 ; \mathrm{f}_{2}$ frequencies $1-8 \mathrm{kHz}$ ).

DPOAE measurements have long been employed in clinical practice to distinguish between normal and impaired hearing, and more specifically, cochlear function (e.g., [25]). DPOAEs have been leveraged for ototoxic monitoring approaches such that DPOAE measurements consistently demonstrate earlier signs of ototoxic damage at the same frequencies when compared to conventional $(1-8 \mathrm{kHz})$ behavioral hearing thresholds (e.g., $[2,7,26])$. It has also been demonstrated that higher frequency $(>8 \mathrm{kHz})$ information is most valuable for timely detection of cochlear dysfunction and specifically utilizing high-frequency DPOAEs [16,27]. Furthermore, the feasibility of measuring DPOAEs beyond $8 \mathrm{kHz}$ has been demonstrated across a range of ages (10 to 65 years) with normal-hearing [21] as well as repeatable over time in healthy, young, normal-hearing adults and in a patient population not exposed to ototoxic medications during monitoring [28-30].

Studies have demonstrated that higher frequency (up to $16 \mathrm{kHz}$ ) DPOAE measurements can provide more insight into changes in cochlear physiology that may be utilized in clinical management [21]. Clinically, at frequencies of $\leq 8 \mathrm{kHz}$, DPOAEs are measured by changing the frequencies of $f_{1}$ and $f_{2}$, while keeping the ratio between them and the levels $\left(L_{1}\right.$ and $\left.L_{2}\right)$ constant and recording the level of the response (in $\mathrm{dB}$ ). This frequency sweep can be completed across a wide frequency range with greater distance between frequencies tested (commonly referred to as a "gross" frequency sweep) or across a more concentrated frequency range with smaller distances between frequencies tested (commonly referred to as a "concentrated" frequency sweep) [31]. There is the possibility that DPOAEs at lower frequencies $(\leq 8 \mathrm{kHz})$ have characteristics that differ from those at higher frequencies due to differences in the underlying physiology at the base and apex of the cochlea (see review [32]). However, a study by Dreisbach and Siegel [28] demonstrated that DPOAEs measured at high frequencies were comparable up to about $10 \mathrm{kHz}$ as those measured in the lower frequency range. That said, DPOAEs at higher frequencies, while still measurable, were lower in level than those at $10 \mathrm{kHz}$ and below [28]. Although not quite as repeatable as in the lower frequencies $(\leq 8 \mathrm{kHz})$, high-frequency DPOAE frequency sweeps have been shown to be repeatable in normal-hearing adults [29] and a patient population not receiving ototoxic medications during monitoring [30]. This suggests that high-frequency DPOAEs are potentially a very powerful tool for ototoxic monitoring.

Although frequency sweep DPOAEs are the most often used, and can be measured reliably at high frequencies, there are two other DPOAE paradigms that could provide a more sensitive look into potential damage to the cochlea as a result of ototoxic medications. Specifically, these paradigms are stimulus level sweeps and ratio sweeps which are used to determine detection thresholds and group delay values, respectively $[7,21,28,31,33]$. Currently, DPOAE stimulus level and ratio sweeps have predominantly been used for research and have not translated to regular clinical practice. However, there is evidence that signs of ototoxic damage may be demonstrated with the use of advanced DPOAE techniques before the traditional frequency sweep at frequencies $<8 \mathrm{kHz}$ [33].

In the case of level sweeps, $\mathrm{f}_{1}$ and $\mathrm{f}_{2}$ frequencies are held constant, as is the ratio between them, and stimulus level is increased until a measurable DPOAE is recorded. Level sweeps are recorded 
as input-output (I/O) functions, which are examined to determine a detection threshold. A detection threshold can be defined as the first level of the stimulus that produces a measurable DPOAE (typically, $6 \mathrm{~dB}$ signal-to-noise ratio or SNR) and results in continued growth as stimulus levels are further increased. Increasing the stimulus level can be done by increasing the levels of the two tones, $\mathrm{L}_{1}$ and $\mathrm{L}_{2}$, together (either $\mathrm{L}_{1}=\mathrm{L}_{2}$ or when the difference between $\mathrm{L}_{1}$ and $\mathrm{L}_{2}$ is consistent), or by holding one constant and increasing the other until a present DPOAE is observed [31]. In general, holding the $\mathrm{L}_{2}$ constant and increasing the $\mathrm{L}_{1}$ shows greater growth and overall larger DPOAE levels are measured when $L_{1}$ is greater than $L_{2}$ regardless of which level is varied $[28,31]$. In a study of patients receiving tobramycin (an ototoxic aminoglycoside antibiotic) where detection thresholds for frequencies $\leq 8 \mathrm{kHz}$ were examined, changes in DPOAE detection threshold were observed before clinically significant changes in the audiometric thresholds as well as before changes in the DPOAE frequency sweep paradigm (current clinical standard) [33]. In this study, detection thresholds were examined at $\mathrm{f}_{2}$ frequencies of 4.3 and $8.5 \mathrm{kHz}$ and changes in the higher of the two frequencies occurred sooner than changes at the lower frequency for patients receiving antibiotics. Additionally, all subjects that received any dose of tobramycin demonstrated elevated DPOAE detection thresholds when compared to the healthy control subjects [33] suggesting that detection thresholds are sensitive to even slight ototoxic damage. This study highlights that DPOAE detection thresholds may be more useful for monitoring the first signs of change within the cochlea as a result of ototoxic damage even at more traditional frequency ranges (closer to $8 \mathrm{kHz}$ ). As mentioned previously, DPOAE detection thresholds vary depending on which stimulus levels are varied (both together, or one constant while the other is varied). Because the study examining detection thresholds for ototoxic monitoring focused on frequencies $\leq 8 \mathrm{kHz}$, it is important to note that DPOAE detection thresholds at high frequencies have been shown to respond in a similar manner as DPOAEs at frequencies $\leq 8 \mathrm{kHz}[28,31]$. Thus, since changes in detection thresholds as a result of ototoxic damage are seen first in the $8 \mathrm{kHz}$ region when looking at frequencies $\leq 8 \mathrm{kHz}$ and detection thresholds can be measured at higher frequencies, detection thresholds at high frequencies ( $>8 \mathrm{kHz}$ ) may be even more telling of insults to the cochlea as a result of ototoxic damage than high frequency DPOAE frequency sweeps, especially as they have been shown to be repeatable in a patient population [30].

DPOAE ratio sweeps also were sensitive to ototoxic damage [33]. The levels of both stimulus tones $\left(\mathrm{L}_{1}\right.$ and $\left.\mathrm{L}_{2}\right)$ are held constant in this paradigm, as is one of the stimulus tone frequencies $\left(\mathrm{f}_{1}\right.$ or $\mathrm{f}_{2}$ ), while the other stimulus tone is swept across frequencies, changing the ratio between the frequencies $\left(f_{2} / f_{1}\right)$. This paradigm uses the phase of the distortion product $\left(2 f_{1}-f_{2}\right)$ that travels from the cochlea to the ear canal to calculate the travel time of the DPOAE $[21,28,29]$. To calculate a group delay value (referred to as group delay hereafter), the phase of the distortion product is plotted against the frequency of $2 f_{1}-f_{2}$ and the slope of that curve is determined. This paradigm often involves fixing $f_{2}$ and varying $f_{1}[28,29,33]$. In the conventional frequency range for DPOAEs $\leq 8 \mathrm{kHz}$, group delay was more sensitive (by showing change sooner) than high frequency audiometry or frequency sweep DPOAEs at traditional frequencies $(\leq 8 \mathrm{kHz})$ when monitoring patients receiving tobramycin [33]. Additionally, group delay has been shown to be repeatable across testing sessions at high frequencies in normal hearing adult subjects and a patient population $[29,30]$. Of note, the slope of the phase (used to calculate group delay) is not always consistent across frequencies $[28,29,34]$. There is a general trend of shorter group delays/travel times for higher frequencies [28,29]. Thus, norms for group delay will be different at each frequency tested, so comparing across frequency (high to low) is not likely to lead to meaningful assessment of damage and should be considered during analysis of group delays. Although there are variations in group delays across frequencies, the results are repeatable within an individual over time [29,30]. DPOAE group delay results tested at high frequencies, may give an earlier indication of cochlear damage than the traditional means of ototoxic monitoring (high frequency audiometry and DPOAEs $\leq 8 \mathrm{kHz}$ or high frequency DPOAEs with the commonly used frequency sweep paradigm), as has been shown in previous research [33]. Katbamna et al. [33] reported prolonged DPOAE group delays in patients with cystic fibrosis $(\mathrm{CF})$ receiving low and low-to-moderate 
cumulative doses of tobramycin regardless of age, but higher doses of tobramycin in older patients (15 to 23 years) resulted in a shorter DPOAE group delays. As DPOAE group delays change (longer or shorter compared to baseline) in the presence of cochlear damage, expectations may depend on the duration of exposure and/or the underlying physiologic consequences of the damage [33,35-37].

Clinical practice may be enhanced by emerging DPOAE applications, including advanced calibration techniques to ensure stability of serial measurements, high-frequency stimuli to assess basal cochlear function, concentrated frequency monitoring protocols, detection thresholds, and group delays to gauge $\mathrm{OHC}$ function. These DPOAE applications may permit visualization and quantification of the earliest signs of underlying cochlear damage which may go undetected with conventional methods. This has the potential to facilitate more meaningful clinical interpretations. In a recent report, high-frequency DPOAE monitoring using frequency, level, and ratio sweeps resulted in clinically significant changes for patients receiving cisplatin, oxaliplatin, and carboplatin [16]. The current paper aims to elaborate on that work by assessing which of the three DPOAE paradigm categories is most sensitive to changes in the auditory system as a result of ototoxic therapies. It is hypothesized that significant changes in detection threshold and/or group delays may be seen before changes in the frequency sweep paradigm based on previous reports [33]. Katbamna et al. [33] reported that changes were seen first for DPOAE thresholds and group delays compared to frequency sweeps in children with cystic fibrosis receiving antibiotic treatments versus controls. In addition, these paradigms have been reported to be repeatable over time and sensitive to platinum exposure $[16,29,30]$. The current report analyzes different DPOAE paradigms used in patients undergoing chemotherapy treatments with platinum derivatives. Individualized measurement protocols were assessed based on the highest frequencies with measurable DPOAEs, allowing exploration of potential opportunities for clinical translation in order to quantify cochlear dysfunction.

\section{Materials and Methods}

\subsection{Patients}

Twenty-three patients, ages 22 to 79 years set to receive cisplatin $(n=4)$, carboplatin $(n=12)$, oxaliplatin $(n=6)$, or one of these three $(n=1)$ as chemotherapy agents were consented to participate. Preliminary exclusion criteria included positive history of radiation therapy to the head, prior chemotherapy treatment, reflectance measures outside normal limits, no response for air-conduction behavioral thresholds from 1-8 kHz, evidence of retrocochlear hearing loss, and absence of measureable DPOAEs between $2-16 \mathrm{kHz}$. Difficulty with accessing patients during active cancer treatments requiring multiple test sessions (at least two) was observed as anticipated. Three patients did not meet the inclusion criteria-including the one patient with the unknown agent received, one patient switched treatment, one patient passed away, and six patients did not have enough data collected (minimally 2 sessions) to evaluate for the purposes of this study. Thus, data from 12 patients, ages 33 to 79 (mean age $=51$ years, $\mathrm{SD}=12$ ) were analyzed in this study (see Table 1 ). All patients had clear ear canals, normal middle ear function, air conduction behavioral thresholds from $1-8 \mathrm{kHz}$, and present DPOAEs minimally at 4 frequencies between $2-16 \mathrm{kHz}$. These criteria were assessed through otoscopy, middle ear reflectance measures, behavioral audiometry, and DPOAE measures. In order to compare changes across sessions, patients needed at least 2 sessions to be included in the analysis. Testing was not completed for every treatment cycle for each patient, so the number of completed treatments that the patient received did not match the number of sessions tested. Data collection and analyses were done in accordance with Institutional Review Board approvals (San Diego State University and University of California, San Diego and Moores Cancer Center at the University of California, San Diego). 
Table 1. Patient number-sorted by age (in years), gender, platinum agent received, cumulative dose, and frequency of highest distortion product otoacoustic emission (DPOAE) present at baseline determined from the gross frequency sweep data for all 12 patients included in this study.

\begin{tabular}{cccccc}
\hline \multicolumn{1}{c}{ Patient } & Gender & Age (years) & Platinum Agent & $\begin{array}{c}\text { Cumulative Dose } \\
(\mathbf{m g})\end{array}$ & $\begin{array}{c}\text { Highest Frequency DPOAE } \\
\mathbf{( k H z )}\end{array}$ \\
\hline 9 & Female & 33 & Carboplatin & 1455 & 16 \\
16 & Female & 41 & Oxaliplatin & 1057 & 13.3 \\
4 & Female & 42 & Oxaliplatin & 873 & 16 \\
18 & Female & 44 & Carboplatin & 3907 & 13 \\
8 & Female & 45 & Carboplatin & 3779 & 10.6 \\
7 & Male & 47 & Oxaliplatin & 1079 & 7.3 \\
1 & Male & 49 & Oxaliplatin & 519 & 6 \\
12 & Male & 55 & Cisplatin & 163 & 9 \\
10 & Female & 56 & Oxaliplatin & 1202 & 8 \\
6 & Female & 59 & Carboplatin & 2412 & 5.3 \\
2 & Male & 61 & Oxaliplatin & 3669 & 3 \\
3 & Female & 79 & Carboplatin & Unknown & \\
\hline
\end{tabular}

\subsection{Equipment, Software, and Calibration}

The emission probe system used had all the capabilities necessary to reliably measure DPOAEs up to $16 \mathrm{kHz}$. Prior to DPOAE measurements, a cavity with acoustic characteristics similar to the human ear canal (B\&K 4157 ear canal simulator) was used to estimate distortion from the transducers and microphones. Distortion was generally $90 \mathrm{~dB}$ lower than the stimulus levels. DPOAEs were recorded in the ear canal in response to two stimulus frequencies $\left(f_{1}\right.$ and $\left.f_{2}\right)$ which were first converted from digital-to-analog (D/A) utilizing a MOTU $96 \mathrm{kHz}$ Audio Firewire Interface (Cambridge, MA, USA) then delivered to the ear canal using modified Audax TW010F1 tweeters. The DPOAE was recorded using an ER 10B+ probe microphone. Emissions were amplified $20 \mathrm{~dB}$ using a pre-amplifier and then converted from analog-to-digital (A/D) by the MOTU interface. The emission was then collected using EMAV (Otoacoustic Emission Averager) software [38] which recorded $46 \mathrm{~ms}$ time windows of the ear canal sound pressure. These recordings were gathered into two interleaved buffers, and each buffer was averaged in the time domain. Fast Fourier transform (FFT) was used to calculate the difference between the two sub-averages $(\mathrm{A}-\mathrm{B})$ to estimate the noise floor. The level and phase of stimulus tones and distortion products in the ear canal were calculated using a grand average $((A+B) / 2)$. Recordings were averaged in total for at least $4 \mathrm{~s}$ until one of two automatic stopping criteria were met: the noise floor at the frequency that corresponded to the DPOAE frequency was less than $-20 \mathrm{~dB}$ SPL, or until $4 \mathrm{~s}$ of recording were averaged with no artifact. Forward pressure level (FPL) calibration was used for more control over stimulus levels to reduce variability and the effect of standing waves in DPOAE measurements, which increased sensitivity and reliability when compared to traditional sound-pressure level (SPL) calibration $[18,19,21]$. The reduction in variability observed across sessions allows for smaller changes due to ototoxicity to be detected in DPOAE measurements. Additionally, calibration files were used to extract reflectance data and compared across sessions to ensure middle ear function was unchanged between sessions. Additional details are provided in Dreisbach et al. [16].

\subsection{Procedures}

Ideally, patients were tested prior to chemotherapy infusions at the beginning of treatment (baseline; trial 1), during treatment, and following treatment (additional tests during or following treatment were performed as the schedule permitted). Baseline assessment was conducted for all participants prior to beginning treatment with the exception of one patient who had baseline testing completed at the first infusion. Due to variance in clinical treatment protocols, testing during treatment took place at different points in the treatment schedule for each patient. All testing was completed 
within approximately three months for each patient with baseline evaluations lasting approximately $2 \mathrm{~h}$ and $1.5 \mathrm{~h}$ for monitoring and post-treatment evaluations. During testing, patients were in a hospital bed either sitting or slightly reclined in a private room (in the infusion center) to reduce environmental noise from interfering with the measurements. The full test battery included middle ear reflectance, pure-tone behavioral hearing thresholds (1-16 kHz; [16]), spontaneous OAEs (i.e., SOAEs), and nine DPOAE paradigms for each session. The modification for monitoring sessions was that hearing thresholds at the 7 highest frequencies eliciting a response at baseline were evaluated [16]. In general, depending on time, cooperation by the patient and nursing staff, and environmental noise, some tests were not completed for some sessions. Prior to each test, patients were given instructions specific for that task. In this manuscript, data from patients with at least two testing sessions for two or more of the nine DPOAE paradigms were analyzed.

Nine DPOAE paradigms were collected, which resulted in 11 analyses (refer to Section 3.4 Summary of Results). The first paradigm was a gross discrete frequency sweep: $f_{2}=16-2 \mathrm{kHz}$; $\mathrm{f}_{2} / \mathrm{f}_{1}=1.2 ; \mathrm{L}_{1} / \mathrm{L}_{2}=62 / 52 \mathrm{~dB}$ FPL where six points per octave were collected with three total octaves swept (18 points). The remaining eight paradigms were concentrated paradigms [21] based on the highest frequency with a present DPOAE from the gross discrete frequency sweep. The first of which were frequency sweeps $(n=2): \mathrm{f}_{2} / \mathrm{f}_{1}=1.2 ; \mathrm{L}_{1} / \mathrm{L}_{2}=62 / 52 \mathrm{~dB}$ FPL (mid-level) and $\mathrm{L}_{1} / \mathrm{L}_{2}=72 / 72 \mathrm{~dB}$ FPL (high level); spanning 2/3 of an octave in $1 / 48$ steps (32 points) starting at the highest frequency with a present DPOAE from the gross sweep. Followed by level sweeps $(n=4): \mathrm{L}_{1}=57, \mathrm{~L}_{2}=17-67 \mathrm{~dB}$ FPL; $\mathrm{L}_{2}=57, \mathrm{~L}_{1}=27-67 \mathrm{~dB}$ FPL (when varied $\mathrm{L}_{1}$ or $\mathrm{L}_{2}$ changed in $3 \mathrm{~dB}$ steps). Each level sweep $\left(\mathrm{L}_{1}\right.$ and $\left.\mathrm{L}_{2}\right)$ was completed at the two highest frequencies from the gross sweep with present DPOAEs. Lastly, ratio sweeps $(n=2)$ were completed $\left(\mathrm{f}_{2} / \mathrm{f}_{1}=1.1-1.25(0.02\right.$ steps $) ; \mathrm{L}_{1} / \mathrm{L}_{2}=62 / 52 \mathrm{~dB}$ FPL $)$ at the two highest frequencies from the gross sweep with present DPOAEs. Level and phase information from each ratio sweep were evaluated separately.

\subsection{Data Analyses}

Recorded DPOAE levels within an individual had to be $6 \mathrm{~dB}$ above the noise floor and greater than $-20 \mathrm{~dB}$ SPL to be included to provide sufficient monitoring capabilities (refer to review by [2]). As a result, there are data points that do not meet inclusion for clinical utility. Significant DPOAE level changes for the frequency sweep DPOAE paradigms (gross, high-level and mid-level concentrated) were defined as at least $\mathrm{a} \pm 6 \mathrm{~dB}$ absolute change from baseline to following sessions in at least two consecutive frequencies [29].

Input-output $(\mathrm{I} / \mathrm{O})$ functions were created with stimulus level on the $\mathrm{x}$-axis and DPOAE level on the $y$-axis for the level sweep paradigms. From this function, DPOAE detection thresholds were determined by finding the first level of the varied stimulus tone $\left(\mathrm{L}_{1}\right.$ or $\left.\mathrm{L}_{2}\right)$ where the DPOAE met the criteria of $6 \mathrm{~dB}$ SNR with an overall level greater than $-20 \mathrm{~dB}$ SPL and continued growth generally was seen with further increases in the stimulus level. It should be noted that at the highest levels of $\mathrm{L}_{2}$ varied with a fixed $L_{1}$, the DPOAE levels have a tendency to plateau and even decline with further increases in stimulus levels. The stimulus levels typically generating the largest DPOAE levels occur when $L_{1}$ is greater than $L_{2}$, but when the stimulus levels are equal the DPOAE level can plateau and saturate [31]. To be considered as a significant change, the detection threshold had to change by $\geq 9 \mathrm{~dB}$ between sessions [30].

For ratio sweeps, where one frequency is held constant $\left(f_{2}\right)$ and the other frequency $\left(f_{1}\right)$ is varied in small step sizes, both the level and phase of the DPOAE can be analyzed. The DPOAE phase can be used to calculate a travel time or group delay for each frequency region tested. Based on the tonotopic organization of the basilar membrane, it is expected that higher frequencies should produce a shorter group delay in comparison to lower frequencies. Ratio sweeps were analyzed in two ways for this study. First, overall DPOAE level across frequency for each ratio within the sweep were analyzed for absolute changes of $\geq 6 \mathrm{~dB}$ between sessions for a single individual. Second, a customized MATLAB program was used to calculate weighted and unweighted group delays using the phase information 
from the ratio sweeps. This approach of calculating and comparing weighted and unweighted group delays addressed potential issues that could arise during phase analysis. The MATLAB program plotted the phase of the DPOAE as a function of frequency for the ratio sweeps and used the slope of the phase to calculate a group delay. Weighted group delays took into account the level of the noise floor and favored the data with lower noise floors and greater overall level. Given similar unweighted and weighted group delays, only the weighted group delays were reported here. Criteria for a significant change in group delay for an individual was defined as an absolute change of $0.87 \mathrm{~ms}$ or more [31]. In total, 11 analyses for the nine DPOAE paradigms described above were completed (refer to Section 3.4 Summary of Results).

\section{Results}

Case illustrations incorporating a range of agents (carboplatin, oxaliplatin, and cisplatin) as well as serial measurements in individuals undergoing chemotherapeutic treatments are highlighted here. This is offered in order to highlight the clinical utility of different DPOAE paradigms for ototoxic monitoring applications. Individualized measurement protocols and analyses are completed to illustrate targeted monitoring approaches compared to broader bandwidth of cochlear function approaches for clinical translation considerations. Of note, current clinical application for ototoxic monitoring compare outcomes from the monitoring visit to the baseline visit to determine clinically significant changes $[3,4]$.

\subsection{Advanced DPOAE Paradigms in Exposed Individuals_Frequency Sweeps}

Three frequency sweep paradigms (gross, mid-level, and high-level concentrated) were collected and a present DPOAE was defined as having a SNR of at least $6 \mathrm{~dB}$ and an overall DPOAE level of at least $-20 \mathrm{~dB}$ SPL. Additionally, a significant change criterion between sessions was defined as a change of $\pm 6 \mathrm{~dB}$ at two or more consecutive frequencies. As some patients had more sessions than others, it should be noted that if significant changes were found between baseline testing and a subsequent test, but the DPOAE level was $< \pm 6 \mathrm{~dB}$ of baseline values at the final testing session, that patient's measurements will still be qualified as a clinically significant change.

Eight out of the $12(66 \%)$ patients had significant changes in their measured DPOAEs for gross discrete frequency sweeps (patients 1, 4, 6, 8, 9, 10, 16 and 18). Patient 4 completed five testing sessions for this paradigm and significant changes between baseline testing and sessions were observed in session three and four, but session five reflected DPOAE levels returning to the baseline level. Defined significant changes between baseline and final test sessions were observed for patients 6,8 , and 10 (session 4 for patients 6 and 8 and session 3 for patient 10, despite showing changes in the second testing session). Interestingly, these three patients also demonstrated significant changes in lower frequency DPOAEs. Patient 9 demonstrated significant changes between baseline and both subsequent sessions, shown in the top panel of Figure 1. Patients 1, 16 and 18 had a significant change from baseline to their second (and final) testing session. Half of the patients showing significant changes received oxaliplatin and the other half received carboplatin. 


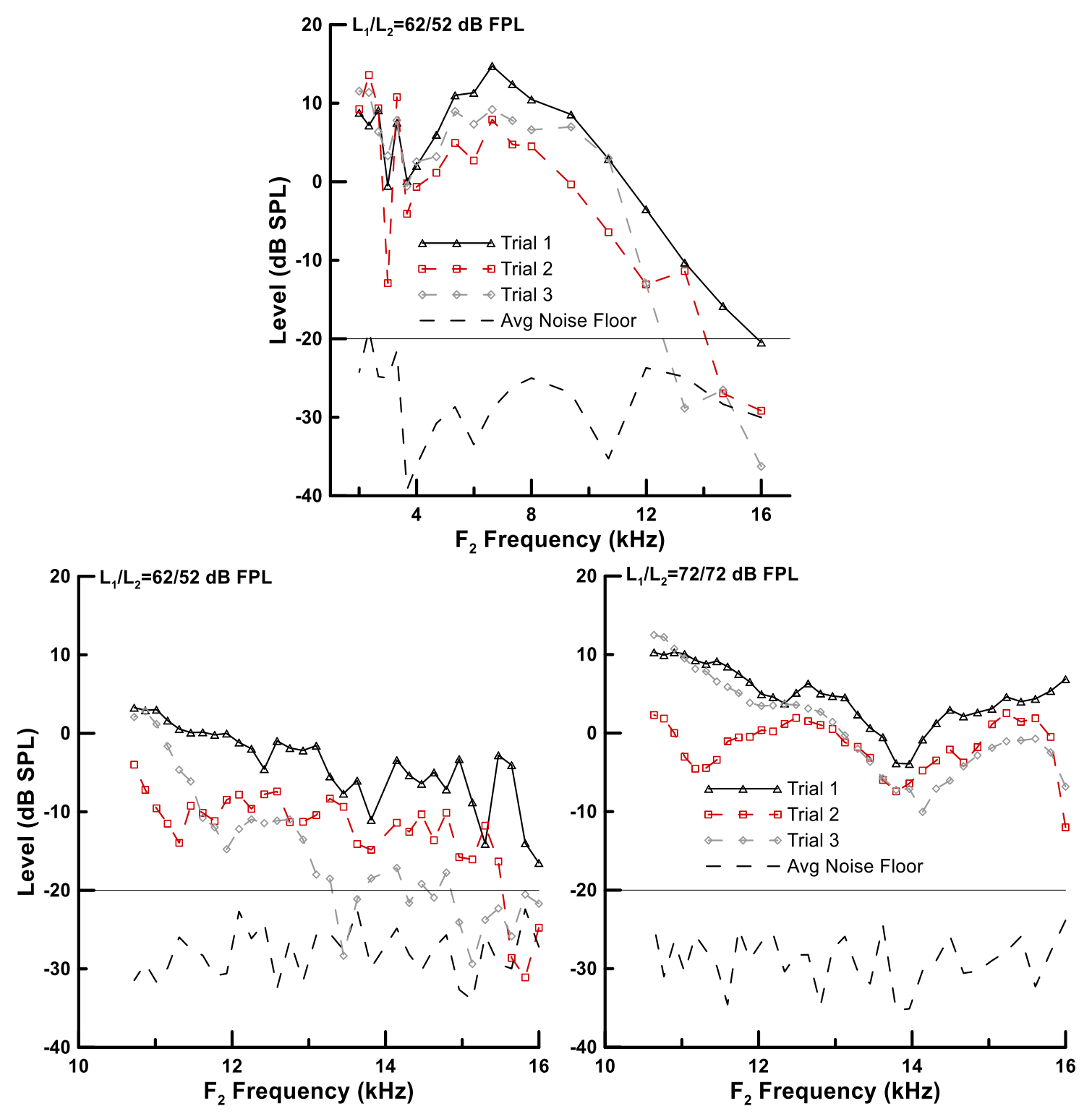

Figure 1. Gross and concentrated frequency sweep results for patient 9. The top panel represents the results from the gross discrete frequency sweep $\left(\mathrm{L}_{1} / \mathrm{L}_{2}=62 / 52 \mathrm{~dB}\right.$ forward pressure level (FPL)), while the bottom left panel illustrates the results from the high-level concentrated frequency sweep $\left(\mathrm{L}_{1}=\mathrm{L}_{2}=72 \mathrm{~dB}\right.$ FPL $)$, and the bottom right panel depicts the data collected for the mid-level concentrated frequency sweeps $\left(\mathrm{L}_{1} / \mathrm{L}_{2}=62 / 52 \mathrm{~dB}\right.$ FPL). Black triangles, red squares, and grey diamonds indicate sessions 1, 2, and 3, respectively. The thin dashed black line indicates the average noise floor for all three sessions. Note, sessions and trials are synonymous.

Eight of the 10 patients $(80 \%)$ demonstrated significant changes in their mid-level $(62 / 52 \mathrm{~dB}$ FPL) concentrated frequency sweep DPOAE levels (patients 1, 4, 6, 8, 9, 10, 16, and 18). Changes in this paradigm for patient 9 are shown in bottom left panel of Figure 1. Where multiple sessions were performed, the frequency ranges showing significant changes were consistent across subsequent sessions for all patients, see Table 2 for details. All of the patients with changes in this paradigm also had changes in the high-level concentrated frequency sweep paradigm, except for patient 6 . All seven patients demonstrated changes for both paradigms in the same general frequency regions; however, the mid-level paradigm had larger ranges of consecutive frequencies with changes. As with the gross frequency sweep results, half of the patients with defined significant changes received oxaliplatin and the other half received carboplatin. 
Table 2. Patient number, platinum agent received, total number of mid-level concentrated frequency sweep sessions performed, sessions (S) where changes occurred, frequency and direction of DPOAE level changes compared to baseline.

\begin{tabular}{|c|c|c|c|c|c|c|}
\hline \multicolumn{7}{|c|}{ Concentrated Frequency Sweep: Mid-Level } \\
\hline Patient & $\begin{array}{l}\text { Platinum } \\
\text { Agent }\end{array}$ & $\begin{array}{l}\text { Number of } \\
\text { Sessions }\end{array}$ & $\begin{array}{c}\text { Highest } \\
\text { Frequency } \\
\text { DPOAE (kHz) }\end{array}$ & $\begin{array}{l}\text { Session of } \\
\text { Change }\end{array}$ & $\begin{array}{l}\text { Frequencies of } \\
\text { Change }(\mathbf{k H z})\end{array}$ & $\begin{array}{c}\text { Direction of } \\
\text { Level Change }\end{array}$ \\
\hline \multirow[b]{2}{*}{1} & \multirow[b]{2}{*}{ Oxaliplatin } & \multirow[b]{2}{*}{2} & \multirow[b]{2}{*}{12} & \multirow[b]{2}{*}{$\mathrm{S} 1$ to $\mathrm{S} 2$} & $7.9-8.0$ & Increase \\
\hline & & & & & $\begin{array}{c}8.4-8.7,9.9-10.0, \& \\
10.3-10.4\end{array}$ & Decrease \\
\hline \multirow{5}{*}{4} & \multirow{5}{*}{ Oxaliplatin } & \multirow{5}{*}{5} & \multirow{5}{*}{16} & $\mathrm{~S} 1$ to $\mathrm{S} 2$ & $10.7-11.5$ & Increase \\
\hline & & & & \multirow{2}{*}{$\mathrm{S} 1$ to $\mathrm{S} 3$} & $10.7-11.0$ & Increase \\
\hline & & & & & $15.3-15.8$ & Decrease \\
\hline & & & & $\mathrm{S} 1$ to $\mathrm{S} 4$ & $10.7-12.3$ & Increase \\
\hline & & & & S1 to S5 & $10.7-11.9 \mathrm{~Hz}$ & Increase \\
\hline \multirow{2}{*}{6} & \multirow{2}{*}{ Carboplatin } & \multirow{2}{*}{4} & \multirow{2}{*}{8} & $\mathrm{~S} 1$ to $\mathrm{S} 2 / \mathrm{S} 3$ & $7.9-8.0 \mathrm{~Hz}$ & \multirow{2}{*}{ Increase } \\
\hline & & & & S1 to $\mathrm{S} 4$ & $7.8-8.0$ & \\
\hline \multirow[b]{2}{*}{8} & \multirow[b]{2}{*}{ Carboplatin } & \multirow[b]{2}{*}{4} & \multirow[b]{2}{*}{10.6} & $\mathrm{~S} 1$ to $\mathrm{S} 2$ & $7.5-7.6 \& 8.5-8.6$ & \multirow[b]{2}{*}{ Decrease } \\
\hline & & & & $\mathrm{S} 1$ to $\mathrm{S} 4$ & $\begin{array}{c}\text { 7.4-7.7, 7.9-8.0, } \\
8.3-9.0, \& \text { 10.3-10.7 }\end{array}$ & \\
\hline \multirow[t]{2}{*}{9} & \multirow[t]{2}{*}{ Carboplatin } & \multirow[t]{2}{*}{3} & \multirow[t]{2}{*}{16} & $\mathrm{~S} 1$ to $\mathrm{S} 2$ & $\begin{array}{l}10.7-10.3,12.6-13.1 \\
14.1-14.3,14.8-15.0 \\
\quad \& 14.6-16.0\end{array}$ & \multirow[t]{2}{*}{ Decrease } \\
\hline & & & & $\mathrm{S} 1$ to $\mathrm{S} 3$ & $11.5-15.8$ & \\
\hline \multirow{2}{*}{$10^{a}$} & \multirow{2}{*}{ Oxaliplatin } & \multirow{2}{*}{3} & \multirow{2}{*}{9} & \multirow{2}{*}{$\mathrm{S} 1$ to $\mathrm{S} 3$} & $6.1-6.5 \& 7.5-7.6$ & Increase \\
\hline & & & & & $8.6-8.7$ & Decrease \\
\hline \multirow{2}{*}{16} & \multirow{2}{*}{ Oxaliplatin } & \multirow{2}{*}{2} & \multirow{2}{*}{13.3} & $C 1+C_{0}$ & $9.4-9.5 \& 9.8-11.1$ & Increase \\
\hline & & & & 51 to 32 & $11.8-11.9$ & Decrease \\
\hline 10 & Carbonlatin & 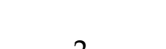 & 12 & $C 1+$ to & $8.0-10.1 \& 10.5-10.6$ & Decrease \\
\hline 18 & Candopiatill & 2 & 13 & S1 to s2 & $11.6-12.0$ & Increase \\
\hline
\end{tabular}

${ }^{\mathrm{a}}$ Indicates no baseline session data, thus $\mathrm{S} 1$ indicates the first session.

Seventy-five percent (9/12 patients) had significant changes in their DPOAE concentrated frequency sweep with high-level stimuli (72/72 dB FPL). Patients 1,2 and 16 had changes from baseline measures to their second (and final) session. Patients 4, 7, 8, 9, 10 and 18 had defined significant changes from baseline to at least one subsequent session and the final session. Results for patient 9 are shown in the bottom right panel of Figure 1. For the patients with changes in subsequent and final testing sessions, the frequency range(s) in which the changes occurred remained the same across sessions for most patients. Note that some patients had more than one frequency range where significant changes occurred. Of the nine patients with significant changes for the high-level concentrated frequency sweep paradigm, six received oxaliplatin $(1,2,4,7,10$, and 16$)$ and three received carboplatin $(8,9$, and 18).

\subsection{Advanced DPOAE Paradigms in Exposed Individuals—Level Sweeps}

As defined in the methods, a detection threshold was identified from level sweep data as the lowest stimulus level that generated a DPOAE (SNR $\geq 6 \mathrm{~dB}$ and overall level $>-20 \mathrm{~dB}$ SPL) with continued growth as the stimulus level was increased further. A significant change criterion from baseline for detection thresholds was defined as a change of greater than $9 \mathrm{~dB}$. Level sweeps were collected at the two highest frequencies generating a DPOAE from the gross frequency sweep. When each frequency was tested, the level of the higher frequency $\left(\mathrm{L}_{2}\right)$ was fixed while the level of the lower frequency $\left(L_{1}\right)$ was varied and vice versa. Ideally, this resulted in four input-output functions for each 
patient. However, due to patient and environment limitations, it was not always possible to run every paradigm in the protocol. Each of the four input-output functions will be described individually.

$L_{2}$ Fixed, $L_{1}$ Varied at Highest Frequency with a Present DPOAE. Four of 10 patients $(40 \%)$ demonstrated significant changes in their detection thresholds when $L_{1}$ was varied and $L_{2}$ was fixed at the highest frequency $\left(f_{2}\right)$ with a present DPOAE. Patients 7,9 , and 10 had a significant increase (worsening) or absence for detection threshold identification at subsequent sessions. Patient 7 had four total sessions with an observed detection threshold only for the first session, while patients 9 and 10 had detection thresholds for their first sessions but not for their final (second) session. Patient 6 had no measurable detection threshold for the first three sessions, but session 4 resulted in an identified detection threshold. Patients 7 and 10 received oxaliplatin and patients 6 , and 9 received carboplatin.

$L_{2}$ Fixed $L_{1}$ Varied at Second Highest Frequency with a Present DPOAE. Six of nine patients (66\%) demonstrated significant changes in identified DPOAE detection thresholds when $\mathrm{L}_{2}$ was held constant and $L_{1}$ was varied at the second highest frequency with a present DPOAE chosen from the gross frequency sweep. Patient 6 had increased (worse) detection thresholds for the second and fourth (final) sessions compared to baseline. Patient 7 demonstrated a worsening of detection threshold at the second session, but the detection threshold returned to its original (baseline) level by the third session and while the threshold worsened by the final session this was not considered a clinically significant change (see Figure 2, left panel). A detection threshold was no longer identified for the second (final) session for patient 9 when a baseline detection threshold was present. Patients 8,16 and 18 all demonstrated significant improvements in their detection thresholds between the baseline session and the final session (patients 16 and 18 had only two sessions). Patient 8 did not have a baseline session for most of the paradigms, including this one, but demonstrated a consistent detection threshold for the first two sessions, followed by a defined significant improvement by the third (final) session. Patients 7 and 16 received oxaliplatin while patients $6,8,9$, and 18 received carboplatin.
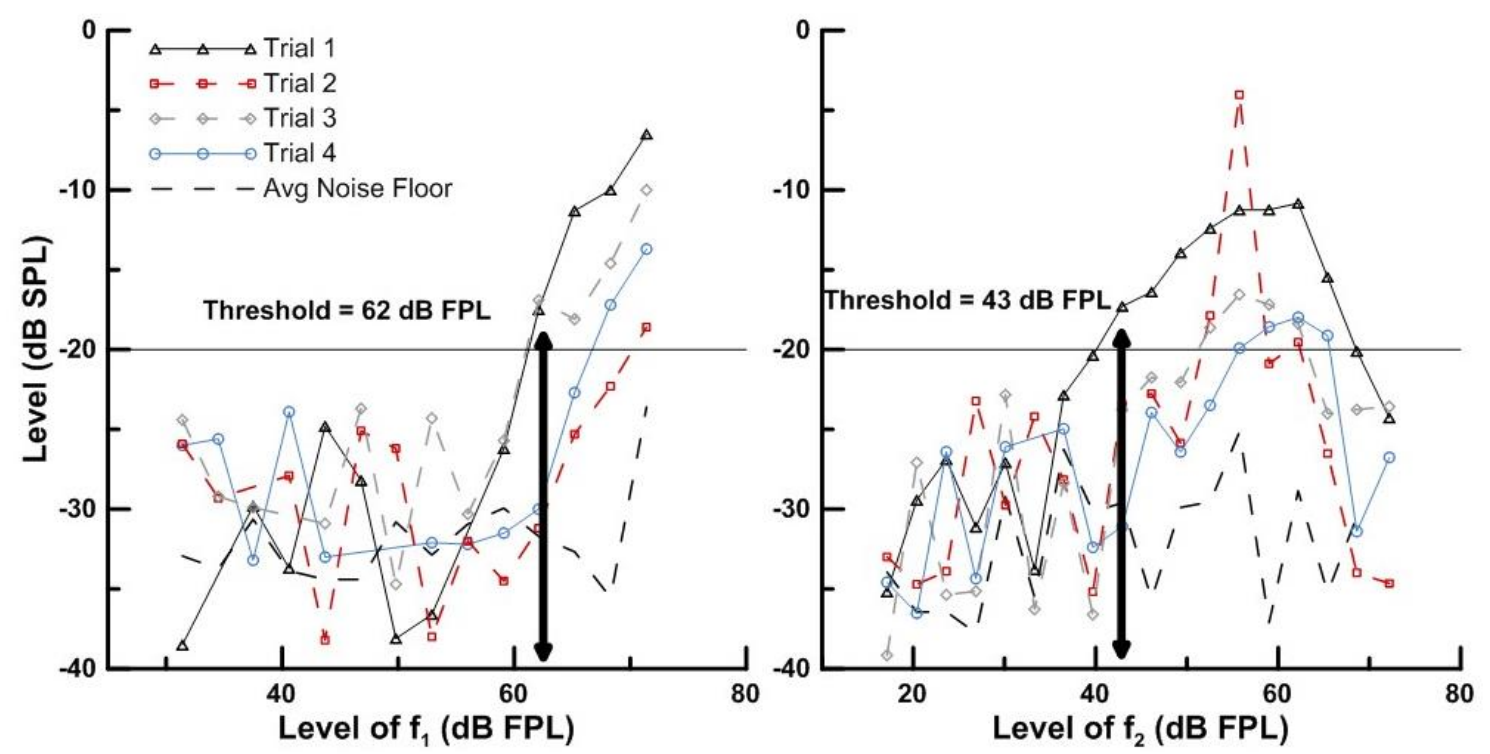

Figure 2. Detection thresholds at the second highest frequency with a present DPOAE (6.6 kHz) for patient 7. The left panel depicts the input-output $(\mathrm{I} / \mathrm{O})$ function when $\mathrm{L}_{1}$ is varied in $3 \mathrm{~dB}$ steps $\left(\mathrm{L}_{2}=57\right.$, $\mathrm{L}_{1}=27-67 \mathrm{~dB}$ FPL $)$ and the right panel shows the results when $\mathrm{L}_{2}$ is varied in $3 \mathrm{~dB}$ steps $\left(\mathrm{L}_{1}=57\right.$, $\mathrm{L}_{2}=17-67 \mathrm{~dB}$ FPL). Black triangles 1, red squares 2, grey diamonds, and blue circles indicate sessions 1 through 4 , respectively. The thin dashed black line indicates the average noise floor for all four sessions. Vertical arrows indicate the detection threshold identified at the baseline session. Sessions and trials are synonymous.

$L_{1}$ Fixed $L_{2}$ Varied at Highest Frequency with a Present DPOAE. Fifty percent of patients (5/10) demonstrated significant changes in DPOAE detection thresholds. Patients 8, 9, 10, 16, and 18 had 
either increased (worsening) detection thresholds or complete absence of a detection threshold at the highest frequency tested at a subsequent session. Furthermore, patients 8 and 10 did not have a baseline test to compare the three subsequent sessions; however, the first session demonstrated a detection threshold that subsequently not observed by the final session ( $>9 \mathrm{~dB}$ shift), resulting in a significant change. Patients 9, 16, and 18 all had a detection threshold at their first session and an absent detection threshold at their second (final) session. Patients 10 and 16 received oxaliplatin and patients 8,9 , and 18 received carboplatin.

$L_{1}$ Fixed, $L_{2}$ Varied at Second Highest Frequency with a Present DPOAE. Nine of the 10 patients $(90 \%)$ demonstrated significant changes in their DPOAE detection thresholds for the $\mathrm{L}_{2}$ sweep at the second highest frequency with a present DPOAE. Patient 4 had a worsening in DPOAE detection threshold from session 1 to sessions 2 through 4 with a return back to baseline by the final session (session 5). Patients 6, 7, 8, 9, and 12 exhibited either a worsening or absence of detection thresholds in subsequent sessions. Patients 6 and 12 had a present detection threshold for the first session, but by the second (final) session the detection threshold had disappeared. Patient 7 had a present detection threshold for session 1, absent detection threshold for session 2, and present but worsened (increased) detection thresholds for sessions 3 and 4 compared to baseline (see Figure 2, right panel). Of note, patient 8 had no baseline testing session, but changes were deemed clinically significant between sessions that were recorded. Patient 9 exhibited an $18 \mathrm{~dB}$ increase (worsening) in detection threshold from session one to the final (second) session. Patients 10,16 and 18 demonstrated improvements in detection thresholds. Although the term improvement is used here, for DPOAE detection threshold, any change (positive or negative) that met the criteria was considered significant and cause for concern. Patient 10 had no measurable detection threshold during the first session, but there was a detection threshold during the subsequent sessions. Patient 16 did not have a measurable detection threshold during baseline testing, but a detection threshold was measured for their second (and last) session. Patient 18 had a present detection threshold for both sessions, but the detection threshold was $24 \mathrm{~dB}$ lower (improved) in the second session compared to baseline. Patients 4, 7, 10 and 16 received oxaliplatin, patients $6,8,9$, and 18 received carboplatin, and patient 12 received cisplatin. Overall, more patients demonstrated significant changes in DPOAE detection thresholds when $\mathrm{L}_{1}$ was fixed and $\mathrm{L}_{2}$ was varied compared to when $\mathrm{L}_{2}$ was fixed and $\mathrm{L}_{1}$ was varied and only one patient demonstrated changes for all four I/O functions (patient 9-carboplatin).

\subsection{Advanced DPOAE Paradigms in Exposed Individuals-Ratio Sweeps}

As outlined in the methods section, ratio sweeps were analyzed in two ways; examination of overall DPOAE level for the varied ratios and calculation of unweighted and weighted group delays. To be considered a significant change, the absolute change in DPOAE level had to be $\geq 6 \mathrm{~dB}$ between sessions for a single individual and a significant change in group delays for an individual was defined as an absolute change of $0.87 \mathrm{~ms}$ or more [30]. As with level sweeps, ratio sweeps were collected at the highest and second highest frequency with a present DPOAE based on the gross discrete frequency sweep, for a total of two ratio sweeps.

DPOAE Levels for Ratio Sweep at Highest Frequency with a present DPOAE. Seven of nine patients $(78 \%)$ demonstrated significant changes in overall DPOAE level for the ratio sweep collected at the highest frequency with a present DPOAE for each individual. Patients 4, 6, 7 and 9 had overall DPOAE changes across all sessions at several consecutive frequencies. Results for patient 9 are seen Figure 3 in the left panel. Patient 16 demonstrated significant changes at two consecutive frequencies from baseline to the second and final session. Similarly, patient 18 had changes in DPOAE level across several consecutive frequencies from baseline to the second (final) session. As with the other paradigms, patient 8 did not have a baseline session, but significant changes were seen from the first testing session to the last testing session at consecutive frequencies, generally at the narrower ratios (when $\mathrm{f}_{2}$ and $\mathrm{f}_{1}$ are more closely spaced). Patients 4,7 , and 16 received oxaliplatin and patients $6,8,9$, and 18 received carboplatin. 

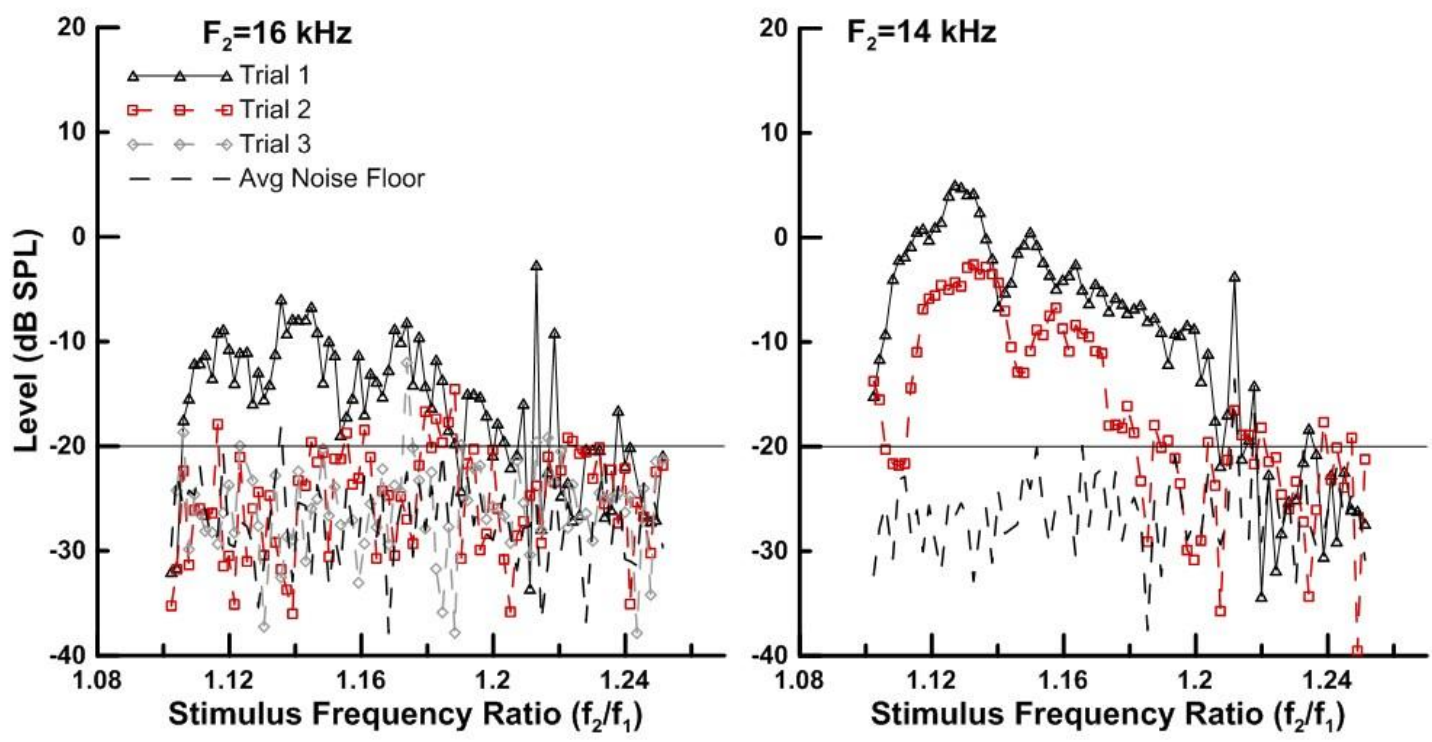

Figure 3. Ratio sweep data $\left(\mathrm{f}_{2} / \mathrm{f}_{1}=1.1-1.25\right.$ (0.002 steps), $\mathrm{L}_{1} / \mathrm{L}_{2}=62 / 52 \mathrm{~dB}$ FPL) for patient 9 at the highest (left panel) and second highest (right panel) frequency with a present DPOAE from the gross sweep. Black triangles, red squares, and grey diamonds indicate session 1, 2, and 3, respectively. The thin dashed black line indicates the average noise floor for all three sessions. Sessions and trials are synonymous.

DPOAE Levels for Ratio Sweep at Second Highest Frequency with a Present DPOAE. Seven of eight patients $(87 \%)$ demonstrated significant changes in overall DPOAE level for the ratio sweep at the second highest frequency tested with a present DPOAE for each individual. Patient 4 , like many of the other paradigms, had no significant changes from baseline to the final session, but sessions two through four had significant changes that then recovered by the final session. Significant changes in DPOAEs at sessions two through four were typically at narrower ratios. The same trend for this paradigm was observed in patient 6, except with significant changes seen at wider ratios. Patient 8 (no baseline session), had present emissions during the first session but then a portion disappeared for the second session. Absent emissions for the second session were apparent at wider ratios (when there was a larger separation between $\mathrm{f}_{2}$ and $\mathrm{f}_{1}$ ). Patient 9 demonstrated significant changes, both increases and decreases from baseline to the second (final) session at several consecutive frequencies (see Figure 3 , right panel). Patients 7 and 10 had significant changes for at least two consecutive frequencies (ratios); these ratios were mostly in the wider ratio region. Patient 18 demonstrated consistent, significant changes from baseline testing to the second and third sessions; these changes were seen at different consecutive frequency ranges across the full ratio range tested. Patients 4,7 and 10 received oxaliplatin and patients 6, 8 and 18 received carboplatin. Overall, six patients demonstrated significant changes at both the highest and second highest frequency tested.

Group Delay for Highest Frequency with a Present DPOAE. In the weighted group delay examination, three out of nine patients (33\%) demonstrated significant changes in group delays. Changes in group delay between baseline and the second session were not observed for Patient 6 , but group delay significantly decreased by the third (final) session. Patient 7 had a significant reduction in group delay between baseline and the second and third sessions, but by the final session group delay returned to the baseline value, as shown in Figure 4 (right side, $\mathrm{f}_{2}=7 \mathrm{kHz}$ ). Lastly, patient 9 demonstrated a significant decrease in group delays from baseline to the second (final) session. Oxaliplatin was administered to patient 7 while carboplatin was administered to patients 6 and 9 .

In the unweighted group delay examination, three patients out of nine (33\%) had significant changes. The same patients that had significant changes in the weighted group delays also demonstrated significant changes for the unweighted group delays, with similar patterns (patients 6, 7 and 9). 


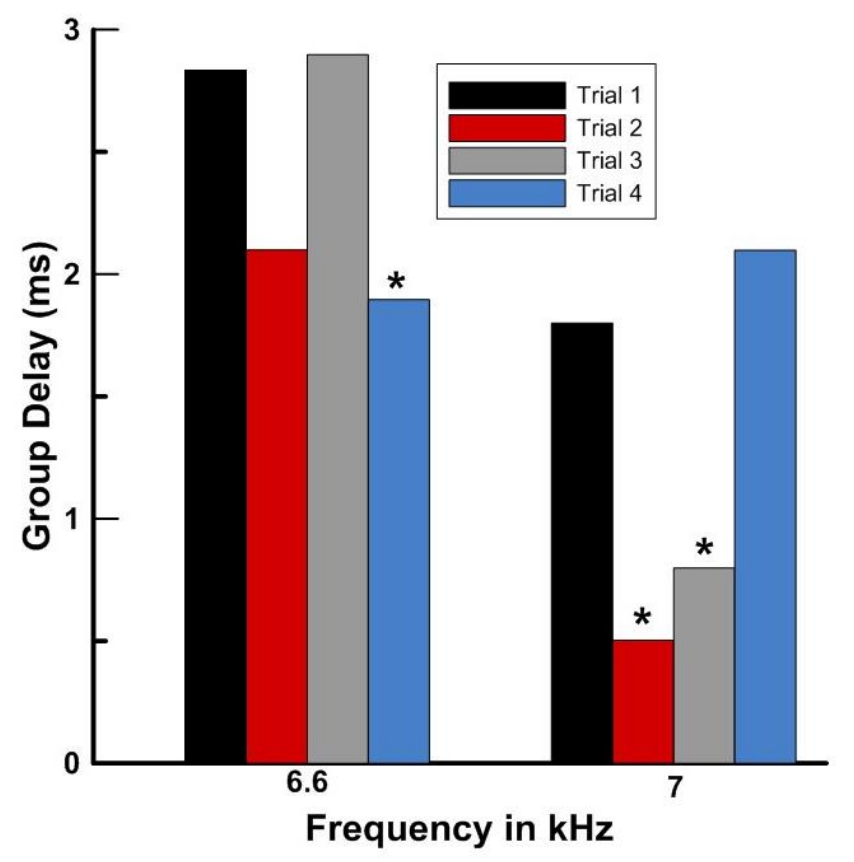

Figure 4. Weighted group delays for patient 7 at the highest (right) and the second highest (left) frequency with present DPOAEs. Black bars indicate session 1, red bars indicate session 2, grey bars indicate session 3, and blue bars indicate session 4 . Session and trial are synonymous. * Indicates defined significant difference from baseline.

Group Delay for Second Highest Frequency with a Present DPOAE. In the case of the calculated weighted group delays, three out of eight patients (37.5\%) had significant changes in group delay. Patient 4 only had an increase in group delay from baseline to the second session, but the value returned to baseline at all subsequent sessions. Patient 7 had a reduction in group delay from session one to session four, with sessions two and three showing no significant changes from baseline, as shown in Figure 4 (left side, $\mathrm{f}_{2}=6.6 \mathrm{kHz}$ ). Patient 18 demonstrated a reduction in group delay from the first to the second (final) session. Patients 4 and 7 received oxaliplatin and patient 18 received carboplatin.

The unweighted group delays analysis, demonstrated significant changes in three out of 8 patients $(37.5 \%)$, both of which also had significant weighted group delay changes. Patient 7 had fluctuations in group delay across the different sessions, with the second session exhibiting a significant change from baseline. However, session three returned to baseline values and the final session had a significant shift from baseline. Patient 10 had an increase in group delay from baseline to both subsequent sessions. Patient 18 had similar changes in unweighted group delay as weighted group delay. Oxaliplatin was received by patients 7 and 10 and carboplatin was received by patient 18 .

\subsection{Summary of Results}

Table 3 provides a summary of the analyses completed, number of patients that had data included in the analyses, which patients demonstrated a defined significant change for the different analyses, and the percentage of significant change for each analysis completed. When monitoring patients, judgement calls had to be made as to how much time would be allowed for the session, based on factors out of the examiner's control (e.g., infusion duration, cooperative nursing staff, health of the patient). For example, if the patient did not have DPOAEs at frequencies $\geq 6 \mathrm{kHz}$, it was assumed that they had hearing loss (confirmed with behavioral threshold results) and it was unlikely that a mid-level concentrated sweep would be completed as the results would provide minimal data points for analysis. Rather, DPOAE level sweeps were completed as these paradigms were the shortest in duration. Conversely, DPOAE ratio sweeps were the last paradigm executed as they were the most time consuming. 
Table 3. Analyses completed $(n=11)$, number of patients tested with each paradigm, number of patients with a defined significant change, and percentage of patients with significant change.

\begin{tabular}{cccc}
\hline \multicolumn{4}{c}{ Summary of Results } \\
\hline Paradigm Analysis & $\begin{array}{c}\text { Patients } \\
\text { Tested }\end{array}$ & $\begin{array}{c}\text { Patients with } \\
\text { Significant Change }\end{array}$ & $\begin{array}{c}\text { Significant Change } \\
\text { Percentage }\end{array}$ \\
\hline Gross Frequency Sweep & 12 & $1,4,6,8,9,10,16,18$ & $8 / 12=66 \%$ \\
\hline Mid-Level Concentrated Sweep & 10 & $1,4,6,8,9,10,16,18$ & $8 / 10=80 \%$ \\
\hline High-Level Concentrated Sweep & 12 & $1,2,4,7,8,9,10,16,18$ & $9 / 12=75 \%$ \\
\hline $\mathrm{L}_{2}$ Fixed, $\mathrm{L}_{1}$ Varied at Highest Frequency & 10 & $6,7,9,10$ & $4 / 10=40 \%$ \\
\hline $\mathrm{L}_{2}$ Fixed, $\mathrm{L}_{1}$ Varied at 2nd Highest Frequency & 9 & $6,7,8,9,16,18$ & $6 / 9=66 \%$ \\
\hline $\mathrm{L}_{1}$ Fixed, L 2 Varied at Highest Frequency & 10 & $8,9,10,16,18$ & $5 / 10=50 \%$ \\
\hline $\mathrm{L}_{1}$ Fixed, L 2 Varied at 2nd Highest Frequency & 10 & $4,6,7,8,9,10,12,16,18$ & $9 / 10=90 \%$ \\
\hline DP Level across Ratio at Highest Frequency & 9 & $4,6,7,8,9,16,18$ & $7 / 9=78 \%$ \\
\hline DP Level across Ratio at 2nd Highest Frequency & 8 & $4,6,7,8,9,10,18$ & $7 / 8=87 \%$ \\
\hline Group Delays at Highest Frequency & 9 & $6,7,9 / / 6,7,9$ & $3 / 9=33 \%$ \\
\hline Group Delays at 2nd Highest Frequency & 8 & $4,7,18 / / 7,10,18$ & $3 / 8=37.5 \%$ \\
\hline
\end{tabular}

\section{Discussion}

In this paper we build on the current knowledge of DPOAE serial measurements by exploring DPOAE measurements at conventional frequencies $(1-8 \mathrm{kHz})$ and high-frequencies $(>8 \mathrm{kHz})$ to capture the earliest changes to cochlear function in individuals undergoing platin-based treatments. Platinum derivatives, such as cisplatin, oxaliplatin, and carboplatin, are often used as treatment for cancer, and are known to cause damage to the auditory system impacting the outer hair cells (OHCs) in the basal end of the cochlea first. Several studies have examined the repeatability of high frequency DPOAEs as well as the sensitivity of behavioral and physiologic measures of auditory function in response to such ototoxic damage. The clinical utility of high-frequency DPOAEs using suitable hardware, calibration, and signal delivery have been accomplished (e.g., [39]) and are further highlighted here through clinical case studies. Most notably, here we explore the influence of platinum derivatives on high frequency DPOAE paradigms in 12 individuals undergoing chemotherapy. DPOAE paradigms included gross $\left(\mathrm{f}_{2}=16-2 \mathrm{kHz} ; \mathrm{f}_{2} / \mathrm{f}_{1}=1.2 ; \mathrm{L}_{1} / \mathrm{L}_{2}=62 / 52 \mathrm{~dB} \mathrm{FPL}\right)$ and concentrated $\left(\mathrm{f}_{2}=\right.$ highest frequency; $\mathrm{f}_{2} / \mathrm{f}_{1}=1.2$; $\mathrm{L}_{1} / \mathrm{L}_{2}=62 / 52$ and $\mathrm{L}_{1} / \mathrm{L}_{2}=72 / 72 \mathrm{~dB}$ FPL $)$ frequency sweeps, level sweeps $\left(\mathrm{L}_{2}=57, \mathrm{~L}_{1}=27-67 \mathrm{~dB}\right.$ FPL and $\mathrm{L}_{1}=57, \mathrm{~L}_{2}=12-67 \mathrm{~dB}$ FPL), ratio sweeps $\left(\mathrm{f}_{2} / \mathrm{f}_{1}=1.1-1.25 ; \mathrm{L}_{1} / \mathrm{L}_{2}=62 / 52 \mathrm{~dB}\right.$ FPL).

Moreover, this paper aims to explore how clinical practice for ototoxic monitoring may be enhanced by emerging DPOAE applications, including use of high-frequency stimuli to assess basal cochlear function, advanced calibration techniques to ensure stability of serial measurements, and targeted monitoring protocols to gauge $\mathrm{OHC}$ function. These DPOAE applications permit visualization and quantification of the earliest signs of underlying cochlear damage which may go undetected with conventional methods (behavioral hearing thresholds as well as standard DPOAE measures up to $8 \mathrm{kHz})$.

\subsection{Serial Measurements of DPOAEs $\leq 8 \mathrm{kHz}$}

DPOAEs elicited with standard parameters $\left(\mathrm{L}_{1} / \mathrm{L}_{2}=65 / 55 \mathrm{~dB}\right.$ SPL; $\left.\mathrm{f}_{2} / \mathrm{f}_{1}=1.22\right)$ at stimulus frequencies $\leq 8 \mathrm{kHz}$ are routinely measured with clinical equipment as part of ototoxic monitoring protocols at baseline with subsequent outcomes compared to baseline results for the purpose of monitoring (reviewed by [5]). DPOAEs, commonly measured at a few points per octave (gross sweep), provide a generally frequency-specific snapshot of overall OHC function to assist with the determination of cochlear dysfunction during monitoring. Figure 5 (left panel) provides a clinical 
case illustration of this "clinical approach" in a 55-year-old individual undergoing cisplatin therapy (100 $\mathrm{mg} / \mathrm{m}^{2}$ per dose) and concurrent radiation therapy to the head and neck (2 Gy per dose). Results in the left panel of Figure 5 illustrate DPOAE levels as a function of frequency ( $\leq 8 \mathrm{kHz})$ across 3 trials (approximately 3 weeks apart) obtained on clinical OAE (Biologic Scout) equipment using traditional SPL calibration and standard stimulus parameters $\left(\mathrm{L}_{1} / \mathrm{L}_{2}=65 / 55 \mathrm{~dB} \mathrm{SPL} ; \mathrm{f}_{2} / \mathrm{f}_{1}=1.22 ; \mathrm{f}_{2}=1.5-10 \mathrm{kHz}\right.$; 4 points per octave).

Clinical Approach

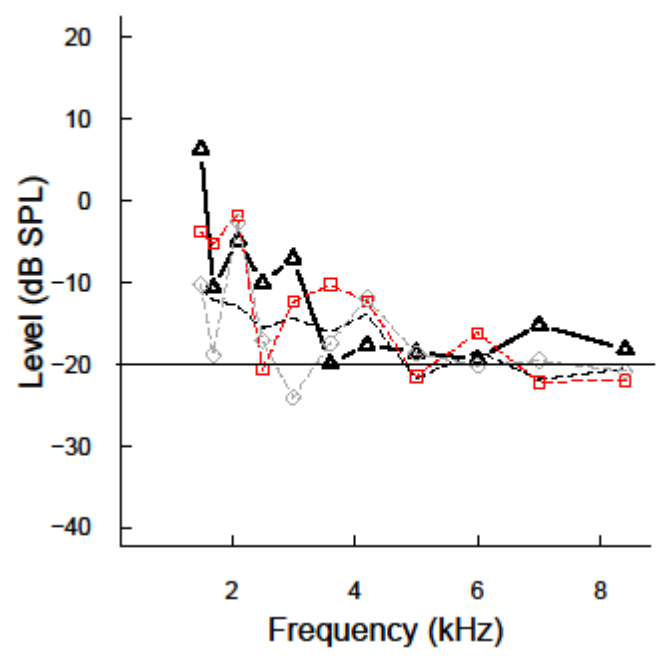

Research Approach

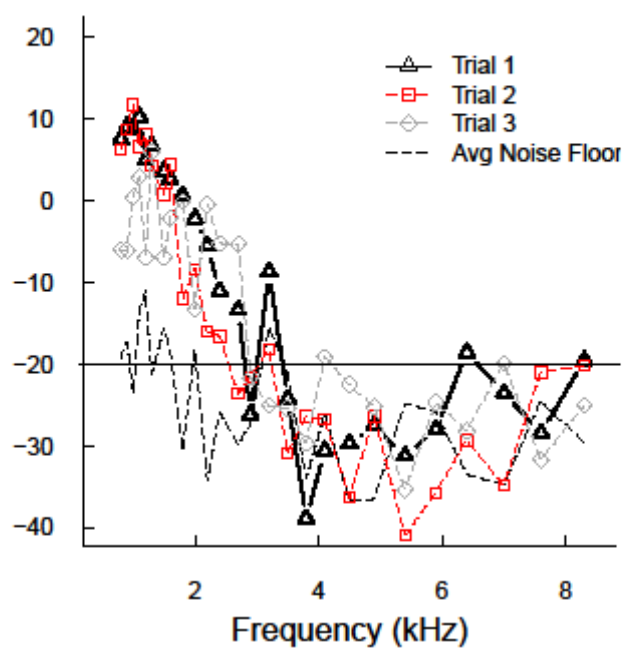

Figure 5. Case illustration of an adult undergoing cisplatin therapy with serial measurement of DPOAEs over 3 trials, averaging 3 weeks apart displayed. DPOAE levels across $f_{2}$ frequencies (up to $8 \mathrm{kHz}$ ) using standard stimulus parameters (1.22; 65/55 dB SPL) tested using a clinical approach (left panel; SPL calibration; 4 points per octave) and research approach (right panel; FPL calibration; $\sim 8$ points per octave). The solid lines with black triangles represent trial 1 (baseline) prior to exposures and the long-dashed lines with red squares or gray diamonds represent trials 2 and 3 (monitoring visits), respectively. The short-dashed lines without symbols are the average noise floor for all trials for the respective approach. The line at $-20 \mathrm{~dB}$ SPL plotted as a reference for visual comparison.

DPOAE levels and average noise floor as a function of frequency $(\leq 8 \mathrm{kHz})$ across 3 trials in the right panel of Figure 5 reflects results obtained with research equipment [21,39] using FPL calibration (see [18-20]). In each panel, the solid and long-dashed lines with symbols represent trials 1-3 with trial 1 noted as the "baseline" measurement prior to the start of treatment. The short-dashed lines without symbols denote the average noise floor across the trials. All DPOAE levels are displayed here for the patient; however, to be considered present for clinical monitoring purposes DPOAEs had to have an SNR of $6 \mathrm{~dB}$, a level greater than $-20 \mathrm{~dB}$ SPL, and have at least two adjacent data points meeting this criterion so not every data point has clinical utility for monitoring. At baseline (trial 1), the clinical approach yielded 3 data points compared to the research approach with 14 data points at frequencies up to $3 \mathrm{kHz}$ for monitoring. The absent responses at higher frequencies are consistent with the pre-existing high-frequency, age-related hearing loss in both ears at trial 1 . This difference in the amount of points available at baseline for continued monitoring is a practical and critical point for the consideration of having an objective, stable DPOAE measurement over time for the purpose of monitoring the pattern of progression of basal cochlear dysfunction (or preservation of cochlear function) throughout treatment.

When DPOAEs are recorded using more closely spaced stimulus frequencies, as in the case of the concentrated sweep for the research approach at $\sim 1 / 8$ th octave steps, a more detailed pattern of minima and maxima of DPOAE levels are revealed. As illustrated in Figure 5, we see that the more concentrated paradigm permits greater characterization of cochlear function as well as more data points to monitor 
from baseline in the standard frequency range. This reflects a paradigm that can be readily employed on standard clinical equipment used in practices today. Moreover, considerable attention in terms of potential clinical utility has been given to even finer frequency resolution measurements, known as fine structure which is understood to be an interference pattern between multiple components of the DPOAE at $2 \mathrm{f}_{1}-\mathrm{f}_{2}$ (e.g., [40-42]). DPOAE fine structure characterization has demonstrated little clinical utility in a population of 10- to 65-year-olds [21]. This highlights that there is an optimal configuration, likely defined by the suspected damage pattern, for what stimulus parameters hold the most clinical utility.

When present, DPOAE levels were larger for the research approach in alignment with improved hardware, calibration and signal delivery capabilities highlighted in previous work [21,39]. Most striking is the comparison between the two approaches for the average noise floors. These are distinctly different with a notably lower noise floor $(-20$ to $-30 \mathrm{~dB})$ for the research approach compared to the clinical approach $(-10$ to -20$)$. It should be noted that the average noise floor was higher at the lower frequencies for both calibration methods and approaches. This lower noise floor in the research approach permits the opportunity to expand SNR criterion for monitoring purposes. Furthermore, the ability to measure DPOAEs at baseline with the research approach permits an illustration (Figure 5) of the value of quantifying stable cochlear health in a region. Specifically, stable DPOAE levels are demonstrated $0.8-1.8 \mathrm{kHz}$ for the research approach relative to baseline.

\subsection{Serial Measurement of DPOAEs with and without Platinum Derivatives}

The need for specialized hardware and calibration techniques complicates the delivery and recording of acoustic stimuli at high frequencies $(\geq 8 \mathrm{kHz})$ in daily clinical practice where access to these capabilities are not commonplace or time- or cost-prohibited. Using customized equipment, high-frequency $(>8 \mathrm{kHz})$ DPOAEs have been measured $[21,28]$ and are repeatable across testing sessions in normal-hearing young adults and children $[28,29,43,44]$, in those with cystic fibrosis tested between ototoxic treatments [32], and identified the earliest signs of damage in adults exposed to ototoxic agents [16]. Traditional calibration methods do not account for individual differences in ear canal geometry and acoustics impacting serial measurement comparisons, while more current in-the-ear calibration techniques attempt to overcome these individual differences through delivery of the signal at the desired level to each individual's eardrum (see reviews by [18-20]). Calibration errors due to standing waves are greatest above $2 \mathrm{kHz}$ (particularly between 3-7 kHz in adults) [28] and applying a FPL calibration approach has demonstrated reduced variability compared to traditional calibration methods and better control of the delivery of stimulus levels at the eardrum [18-20]. Reduced variability across trials hold potential for more sensitive and reliable detection of the smallest changes due to ototoxicity (e.g., [2]).

Figure 6 highlights a case illustration of the extension of DPOAE measures into the upper limit of human hearing for an individual undergoing cisplatin chemotherapy compared to an age-matched control subject to highlight serial measurement variability. As with any clinical tool, the utility depends in part on the variability of the measurement, which in the case of DPOAEs can differ based on a variety of factors (e.g., patient population, equipment used, probe placement, test location and method, stimulus parameters, middle ear status, tester, etc.). For monitoring purposes, several DPOAE measurements are made over time and compared to a baseline measure to determine a clinically significant change. To be considered clinically significant, DPOAE level differences from baseline must exceed test-retest variability making it critical to minimize the variability of controllable factors such as hardware, calibration technique, and test environment [2]. Any successful measure for ototoxicity monitoring needs to be consistent over time in the absence of pathology, so that the effects of the pathology can be confidently segregated from random fluctuation. DPOAEs at conventional frequencies have been found to be repeatable over short (days) and long (weeks) periods of time, with changes in level of 4-9 dB considered significant [45-47]. Repeated measures of DPOAE level are slightly more variable at frequencies above $8 \mathrm{kHz}$ than below in normal-hearing individuals [29] as 
well as patients [16] due in at least part to accurate stimulus control at high frequencies described above. In Figure 6, reduced variability across trials $1-3$ is noted in the control subject (right side) compared to the exposed patient. Test-retest differences across weeks were on the order of $<3 \mathrm{~dB}$ for frequencies up to $20 \mathrm{kHz}$ in the control subject permitting clinically significant changes for the exposed individual to be interpreted as $>3 \mathrm{~dB}$ difference from baseline at two adjacent frequencies as opposed to $>6 \mathrm{~dB}$ difference permitting earlier detection of change. To this end, 6 frequencies at trial 2 (compared to 3 with the $6 \mathrm{~dB}$ criterion) and 7 frequencies at trial 3 (compared to 5 with $6 \mathrm{~dB}$ criterion) were available for comparison to baseline results. Overall, repeatable measures of DPOAEs at high frequencies are feasible and hold clinical utility for improving ototoxic monitoring.

Exposed

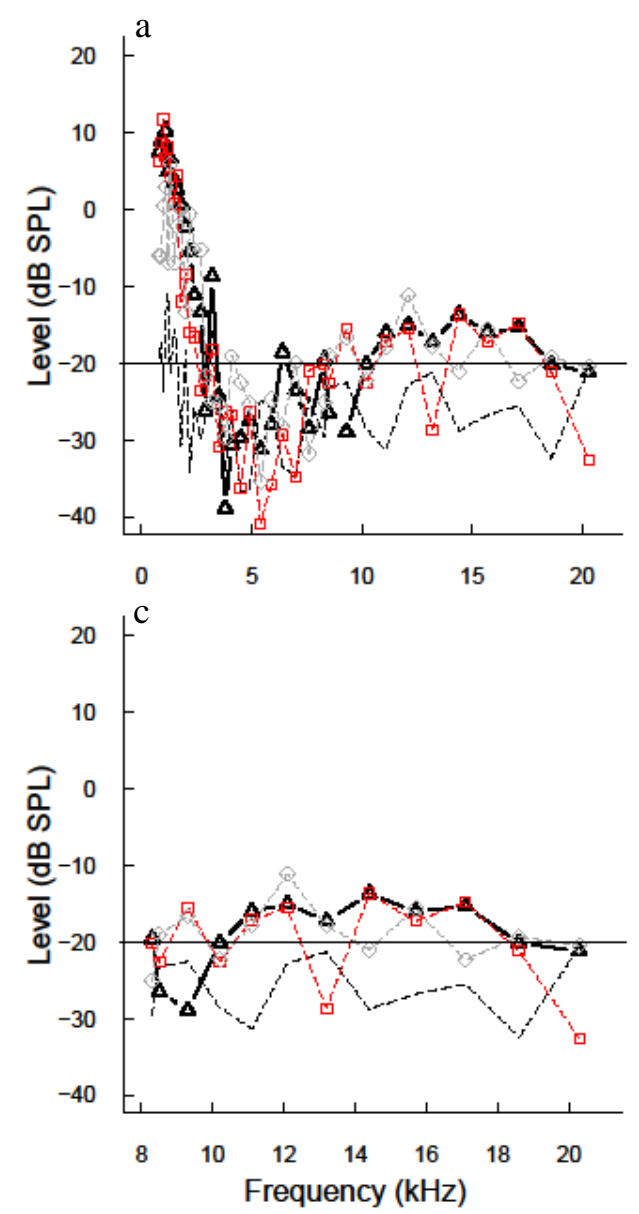

Control
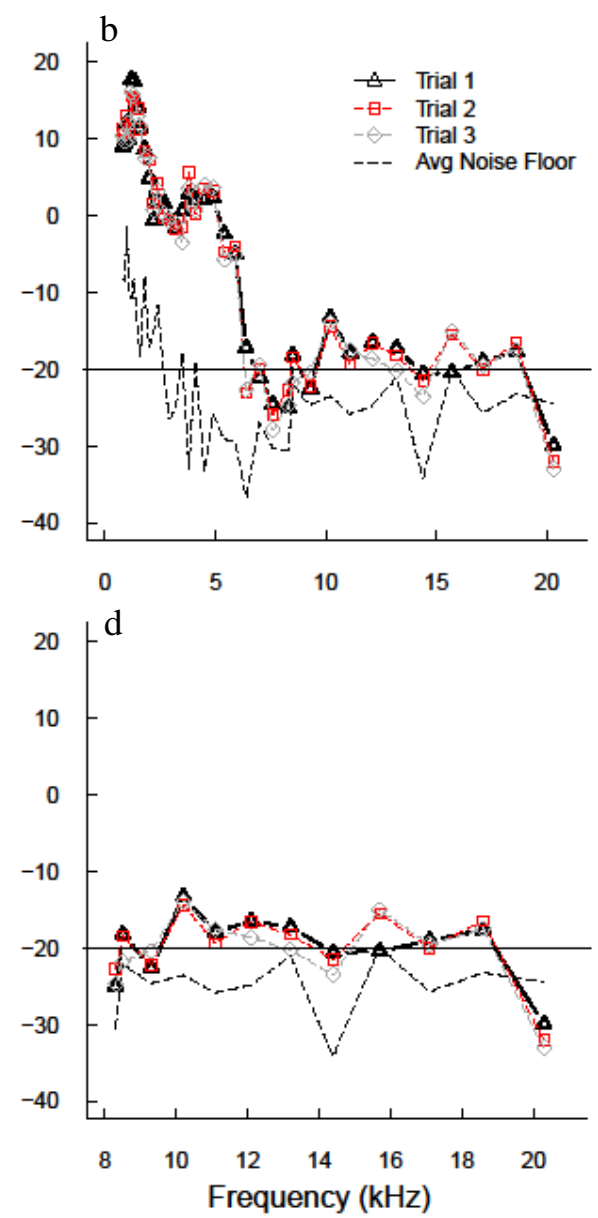

Figure 6. Case illustration of an adult undergoing cisplatin therapy (Exposed; left; a; c; same patient as Figure 5) compared to age-matched control subject (Control; right; b; d) for serial measurement of DPOAEs over 3 trials, averaging 3 weeks apart. DPOAE levels recorded for full bandwidth of $f_{2}$ frequencies ( 0.8 to $20 \mathrm{kHz} ; 1 / 8$ octave step; $1.22 ; 62 / 52 \mathrm{~dB}$ FPL; top; $\mathrm{a}$; $\mathrm{b}$ ) versus limited bandwidth ( 8 to $20 \mathrm{kHz}$; bottom; c; d) using standard stimulus parameters. The solid lines with black triangles represent trial 1 (baseline) prior to exposures and the long-dashed lines with red squares or gray diamonds represent trials 2 and 3 (monitoring visits), respectively, for the exposed individual with trials 1-3 for the control individual matched for testing in chronological time. The short-dashed lines without symbols are the average noise floor for all trials for the respective individual. The line at $-20 \mathrm{~dB}$ SPL plotted as a reference for visual comparison.

\subsection{Advanced DPOAE Paradigm Comparisons (Frequency, Level, and Ratio Sweeps-Group Delays)}

In this paper, we demonstrate the clinical applicability of advanced DPOAE measurement hardware and paradigms. It is all too common that data obtained on typical clinical equipment results 
in very limited (or no) information at baseline in "real world" applications in adult patients. This is a challenge when baseline information is necessary to monitor for the care of the patient. Employing advanced hardware and calibration techniques permitting higher frequency and higher intensity stimuli to be delivered permits a broader range of clinical information than traditional approaches.

Results support ototoxic changes can be detected and monitored successfully by utilizing DPOAE measurements $>8 \mathrm{kHz}$ when suitable hardware/signal delivery capabilities, calibration techniques, and stimulus parameters are employed $[16,21,28-31,48]$. Here, we report clinically-defined significant changes that were often noted at frequencies higher than conventionally tested. When examining the patient data presented in the Results section (refer to Table 3), no significant change for any paradigm tested during serial monitoring was demonstrated for one case (patient 3). Upon further investigation, this patient was the oldest, only had measurable DPOAEs out to $3 \mathrm{kHz}$ for monitoring, and passed away after the second monitoring session. It is unknown if significant changes would have occurred had monitoring continued. Only two monitoring sessions were obtained for patient 12 before passing away. The only significant change for patient 12 with measurable DPOAEs out to $6 \mathrm{kHz}$ occurred in the $\mathrm{L}_{1}$ fixed and $\mathrm{L}_{2}$ varied paradigm at the second highest frequency monitored. Patient 2 only had one significant change, which occurred in the high-level concentrated sweep paradigm. The highest DPOAE frequency for monitoring was $5.3 \mathrm{kHz}$ and only two sessions were completed for patient 2. All other patients $(n=9)$ had a minimum of three significant changes for the analyses completed $(n=11)$ and one patient (9) had significant changes in all analyses, with the exception of group delays calculated at the second highest frequency. The percentage of patients with a significant change for the various analyses ranged from $33 \%$ for group delays at the second highest frequency to $90 \%$ for the $L_{1}$ fixed and $L_{2}$ varied at the second highest frequency. The patients that had a significant change in the gross frequency sweep also had a significant change in the mid-level concentrated sweep and given the gross frequency sweep was conducted initially to determine what frequencies could be monitored, it is not clear that measuring the mid-level concentrated sweep was valuable in this patient population. The greatest number of patients demonstrating significant changes occurred for the high-level concentrated sweep and the $L_{1}$ fixed with $L_{2}$ varied at the second highest frequency monitored. Thus, once the highest frequency with a present DPOAE has been determined, it is recommended that monitoring occur using a high-level concentrated sweep and (time-permitting) level and ratio sweeps at the second and possibly third highest frequency identified. It is possible that with the level and ratio sweeps that the highest frequency identified from the gross frequency sweep was essentially at the limit of cochlear function and therefore, unable to be useful for in depth monitoring (level and ratio sweeps).

Although the small sample size presented here warrants further investigation, these results demonstrate that more concentrated frequency sweeps and controlled administration with clinical OAE equipment may be immediately applied to the clinical practice for improved practice. Results indicate that monitoring high-level concentrated frequency sweeps and level sweeps (detection thresholds) yielded the highest percentage of changes in auditory function for patients undergoing platinum derivative treatments. Thus, highlighting that a hypothesis-driven clinical approach for suspected auditory damage patterns leveraging DPOAEs can be used successfully in ototoxicity monitoring programs for characterizing cochlear health.

\subsection{Clinical Implications}

The earliest sign of changes in cochlear function present at the highest frequencies of human hearing reflecting the basal cochlear region. In turn, clinical ototoxic monitoring approaches target measurements of the highest frequencies (above $8 \mathrm{kHz}$ ) for the detection of the earliest changes in hearing. A snapshot or general understanding of hearing or cochlear function as present or absent holds value in many clinical applications; however, this does not offer the most robust clinical utility. The opportunity for the audiologist to have the tools necessary to detect and quantify function in a particular portion of the auditory system or a "region" of dysfunction in the cochlea is critical 
for multi-disciplinary care team management. As assessment techniques advance to identify more specific site-of-lesion and reduced variability of serial measurement, it is anticipated that more refined management and monitoring solutions may be necessary. Characterizing cochlear function at the baseline evaluation in the presence of hearing loss in adult populations is not only valuable, but necessary for clinical monitoring [27]. This pre-existing hearing loss as well as changes in middle ear status can limit the clinical utility of DPOAEs for monitoring. Many other factors may influence the success of DPOAE measurements as well as the ability of the patient to tolerate and time permitted for the evaluation (see overview by [2]). Moreover, a "one-size-fits-all" approach is not ideal given the highly individualized treatment paradigms and varied hypotheses for suspected underlying auditory damage. To this end, results on the individual level are most insightful for clinical implications.

Generally, when decreased DPOAE levels occur during treatment it is at the highest frequencies evaluated resulting in higher clinical utility of monitoring DPOAEs $>8 \mathrm{kHz}$. In other words, the highest frequencies that evoke a valid DPOAE measure at baseline are typically displaying the greatest reductions at monitoring and/or post-treatment evaluations [7,16,27,49]. Decreased DPOAE levels have aligned well with decreased higher frequency hearing and associated presence of cochlear damage; however, there is evidence of enhancements (increased DPOAE level). Traditionally in the clinic, a positive $(+)$ change has been considered a sign of cochlear health (or "recovery"). However, this is complicated to consider as underlying physiological changes and/or damage patterns (e.g., strial dysfunction, oxidative stress, etc.) as inferred by current clinical tools may suggest reversible or "recovered" (or no) damage due to platin-based therapies (see discussion by [3,9]). Specifically, an enhancement in DPOAE level may be suspicious compared to a decreased DPOAE level in the presence of damage. Because ototoxicity impacts the basal end of the cochlea initially, changes may be detected as reductions in DPOAE levels, as well as enhancements in DPOAE levels if basal contributions are physiologically suppressed/damaged. The DPOAE measured in the ear canal is complex, compromising multiple sources (primarily distortion and reflection) as well as basal contributions $[50,51]$. Using suppression techniques to eliminate basal frequency contributions $\left(>f_{2}\right)$ to the overall DPOAE in rabbits, Martin et al. [51] reported enhancements in DPOAE levels measured from apical frequency regions. They applied physiological manipulations (i.e., anesthesia, diuretic administration, eighth nerve section, noise exposure) which led them to suggest that these changes are phenomena of cochlear origin rather than attributing changes to equipment artifact, the acoustic reflex, high-level DPOAE generators, or cochlear efferent activity on the outer hair cells. With more data sets available, enhancements in DPOAE levels occurring in the presence of platinum derivatives in humans is becoming commonplace and expected at frequencies lower than the highest DPOAE frequency recorded $[2,16]$. An example can be seen in Figure 1 where enhancements were seen at the lowest frequencies for the gross frequency sweep for trials 2 and 3 and again at the lowest frequencies for the high-level concentrated frequency sweep for trial 3. Furthermore, enhancements in DPOAE level may be related to changes in fine structure depth that can be observed with detailed frequency DPOAE measures (e.g., [52]). Regardless of the cause for DPOAE level enhancements in the presence of damaging ototoxic agents, one must consider an enhancement as evidence of cochlear damage rather than "recovery" (refer to discussion by $[2,16,49,52])$. Overall, any clinically significant change in DPOAE level, positive or negative, is cause for concern when monitoring cochlear health.

\section{Conclusions}

DPOAEs are measureable and repeatable in an adult patient population undergoing platin-based therapies across a range of frequencies $(0.8$ to $20 \mathrm{kHz})$ and paradigms. This paper highlights how clinical practice may be enriched by emerging DPOAE applications, including the use of high-frequency stimuli to assess basal cochlear function, advanced calibration techniques to ensure stability of serial measurements, and targeted monitoring protocols to gauge outer hair cell function. These DPOAE applications permit visualization and quantification of the earliest signs of underlying cochlear damage which may go undetected with conventional methods [16]. Furthermore, our evidence supports 
a departure from the "one-size-fits-all" approach to DPOAE measurement in the clinic. Most persuasive are the noted enhancements in DPOAE level captured in patients undergoing treatments that may provide insight to the extent of ototoxic damage if further explored. This has the potential to facilitate more meaningful clinical interpretations and proactive monitoring approaches.

Author Contributions: Conceptualization, G.L.P. and L.E.D.; Data curation, G.L.P. and L.E.D.; Formal analysis, G.L.P., B.V. and L.E.D.; Methodology, G.L.P. and L.E.D.; Project administration, G.L.P. and L.E.D.; Writing-original draft, G.L.P., B.V. and L.E.D.; Writing-review and editing, G.L.P., B.V. and L.E.D.

Funding: Data collection was supported by Knowles Hearing Center at Northwestern University and an American-Speech-Language-Hearing Foundation Clinical Research Grant.

Acknowledgments: We are grateful to Jonathan Siegel, Erin Reid, Nichole Suss, and Sumitrajit Dhar for their contributions to this project. Special thanks to Shawn Goodman for his assistance with MATLAB programming for data acquisition for a portion of the results reported here. We thank Michelle Louie, Jason Yang, Karolina Crawford, Joyce Chin, Rachel Smith, and Matt Bishop, former research assistants in the Auditory Physiology and Psychoacoustics Laboratory, for data collection and entry. The authors also wish to thank the many collaborators on the Hearing Assessment Reformulation Project (HARP) for their contributions for paving the way for this work. We acknowledge contributions from the Moores Cancer Center at the University of California, San Diego and University Illinois Hospital and Health Sciences System, Chicago, Illinois. Heartfelt thanks to the patients who participated.

Conflicts of Interest: The authors declare no conflict of interest.

\section{References}

1. Watts, K.L. Ototoxicity: Visualized in Concept Maps. In Seminars in Hearing; Thieme Medical Publishers: Stuttgart, Germany, 2019; Volume 40, pp. 177-187.

2. Konrad-Martin, D.; Poling, G.L.; Dreisbach, L.E.; Reavis, K.M.; McMillan, G.P.; Lapsley Miller, J.A.; Marshall, L. Serial Monitoring of Otoacoustic Emissions in Clinical Trials. Otol. Neurotol. 2016, 37, e286-e294. [CrossRef] [PubMed]

3. American Academy of Audiology. American Academy of Audiology's Position Statement and Practice Guidelines on Ototoxicity Monitoring; American Academy of Audiology: Reston, VA, USA, 2009.

4. American Speech-Language-Hearing Association. Guidelines for the audiologic management of individuals receiving cochleotoxic drug therapy. ASHA 1994, 36 (Suppl. 12), 11-19.

5. Konrad-Martin, D.; Poling, G.L.; Garinis, A.C.; Ortiz, C.E.; Hopper, J.; O'Connell Bennett, K.; Dille, M.F. Applying US national guidelines for ototoxicity monitoring in adult patients: Perspectives on patient populations, service gaps, barriers and solutions. Int. J. Audiol. 2017, 57, S3-S18. [CrossRef] [PubMed]

6. Dreschler, W.A.; van der Hulst, R.J.; Tange, R.A.; Urbanus, N.A. Role of high-frequency audiometry in the early detection of ototoxicity: II. Clinical Aspects. Audiology 1989, 28, 211-220. [CrossRef] [PubMed]

7. Ress, B.D.; Sridhar, K.S.; Balkany, T.J.; Waxman, G.M.; Stagner, B.B.; Lonsbury-Martin, B.L. Effects of cis-platinum chemotherapy on otoacoustic emissions: The development of an objective screening protocol. Third place—Resident Clinical Science Award 1998. Otolaryngol. Head Neck Surg. 1999, 121, 693-701. [CrossRef] [PubMed]

8. Fausti, S.A.; Frey, R.H.; Henry, J.A.; Robertson, P.G.; Hertert, R.S. Portable stimulus generator for obtaining high-frequency (8-14 kHz) auditory brainstem responses. J. Am. Acad. Audiol. 1992, 3, 166-175. [PubMed]

9. Bielefeld, E.C.; Henderson, D. Mechanisms of Cisplatin Ototoxicity and Routes for Intervention. Perspect. Hear. Hear. Disord. Res. Diagn. 2011, 15, 3-14. [CrossRef]

10. Komune, S.; Aakuma, S.; Snow, J.B. Pathophysiology of the ototoxicity of cis-diamminedichloroplatinum. Otolaryngol. Head Neck Surg. 1981, 89, 275-282. [CrossRef]

11. Dammeyer, P.; Hellberg, V.; Wallin, I.; Laurell, G.; Shoshan, M.; Ehrsson, H.; Arnér, E.S.; Kirkegaard, M. Cisplatin and oxaliplatin are toxic to cochlear outer hair cells and both target thioredoxin reductase in organ of Corti cultures. Acta Otolaryngol. 2014, 134, 448-454. [CrossRef]

12. Hofstetter, P.; Ding, D.; Powers, N.; Salvi, R.J. Quantitative relationship of carboplatin dose to magnitude of inner and outer hair cell loss and the reduction in distortion product otoacoustic emission amplitude in chinchillas. Hear. Res. 1997, 112, 199-215. [CrossRef] 
13. Ding, D.L.; Wang, J.; Salvi, R.; Henderson, D.; Hu, B.H.; McFadden, S.L.; Mueller, M. Selective loss of inner hair cells and type-I ganglion neurons in carboplatin-treated chinchillas. Mechanisms of damage and protection. Ann. N. Y. Acad. Sci. 1999, 884, 152-170. [CrossRef]

14. Kraus, K.S.; Ding, D.; Jiang, H.; Kermany, M.H.; Mitra, S.; Salvi, R.J. Up-regulation of GAP-43 in the chinchilla ventral cochlear nucleus after carboplatin-induced hearing loss: Correlations with inner hair cell loss and outer hair cell loss. Hear. Res. 2013, 302, 74-82. [CrossRef]

15. Ding, D.; Allman, B.L.; Salvi, R. Review: Ototoxic characteristics of platinum antitumor drugs. Anat. Rec. 2012, 295, 1851-1867. [CrossRef]

16. Dreisbach, L.; Ho, M.; Reid, E.; Siegel, J. Effects of Oxaliplatin, Carboplatin, and Cisplatin Across Treatment on High-Frequency Objective and Subjective Auditory Measures in Adults. Perspect. ASHA Spec. Interest Groups 2017, 2, 17-36. [CrossRef]

17. Brown, A.M.; McDowell, B.; Forge, A. Acoustic distortion products can be used to monitor the effects of chronic gentamicin treatment. Hear. Res. 1989, 42, 143-156. [CrossRef]

18. Scheperle, R.A.; Neely, S.T.; Kopun, J.G.; Gorga, M.P. Influence of in situ, sound-level calibration on distortion-product otoacoustic emission variability. J. Acoust. Soc. Am. 2008, 124, 288-300. [CrossRef]

19. Scheperle, R.A.; Goodman, S.S.; Neely, S.T. Further assessment of forward pressure level for in situ calibration. J. Acoust. Soc. Am. 2011, 130, 3882-3892. [CrossRef]

20. Souza, N.N.; Dhar, S.; Neely, S.T.; Siegel, J.H. Comparison of nine methods to estimate ear-canal stimulus levels. J. Acoust. Soc. Am. 2014, 136, 1768. [CrossRef]

21. Poling, G.L.; Siegel, J.H.; Lee, J.; Dhar, S. Characteristics of the $2 \mathrm{f}(1)-\mathrm{f}(2)$ distortion product otoacoustic emission in a normal hearing population. J. Acoust. Soc. Am. 2014, 135, 287-299. [CrossRef]

22. Lee, J.; Dhar, S.; Abel, R.; Banakis, R.; Grolley, E.; Lee, J.; Zecker, S.; Siegel, J. Behavioral hearing thresholds between 0.125 and $20 \mathrm{kHz}$ using depth-compensated ear simulator calibration. Ear Hear. 2012, 33, 315-329. [CrossRef]

23. Kemp, D.T. Evidence of mechanical nonlinearity and frequency selective wave amplification in the cochlea. Arch. Oto-Rhino-Laryngol. 1979, 224, 37-45. [CrossRef]

24. Dorn, P.A.; Piskorski, P.; Gorga, M.P.; Neely, S.T.; Keefe, D.H. Predicting audiometric status from distortion product otoacoustic emissions using multivariate analyses. Ear Hear. 1999, 20, 149-163. [CrossRef]

25. Gorga, M.P.; Neely, S.T.; Ohlrich, B.; Hoover, B.; Redner, J.; Peters, J. From laboratory to clinic: A large scale study of distortion product otoacoustic emissions in ears with normal hearing and ears with hearing loss. Ear Hear. 1997, 18, 440-455. [CrossRef]

26. Arnold, D.J.; Lonsbury-Martin, B.L.; Martin, G.K. High-frequency hearing influences lower-frequency distortion-product otoacoustic emissions. Arch. Otolaryngol. Head Neck Surg. 1999, 125, 215-222. [CrossRef]

27. Reavis, K.M.; Phillips, D.S.; Fausti, S.A.; Gordon, J.S.; Helt, W.J.; Wilmington, D.; Bratt, G.W.; Konrad-Martin, D. Factors affecting sensitivity of distortion-product otoacoustic emissions to ototoxic hearing loss. Ear Hear. 2008, 29, 875-893. [CrossRef]

28. Dreisbach, L.E.; Siegel, J.H. Distortion-product otoacoustic emissions measured at high frequencies in humans. J. Acoust. Soc. Am. 2001, 110, 2456-2469. [CrossRef]

29. Dreisbach, L.E.; Long, K.M.; Lees, S.E. Repeatability of high-frequency distortion-product otoacoustic emissions in normal-hearing adults. Ear Hear. 2006, 27, 466-479. [CrossRef]

30. Dreisbach, L.; Zettner, E.; Chang Liu, M.; Meuel Fernhoff, C.; MacPhee, I.; Boothroyd, A. High-Frequency Distortion-Product Otoacoustic Emission Repeatability in a Patient Population. Ear Hear. 2018, 39, 85-100. [CrossRef]

31. Dreisbach, L.E.; Siegel, J.H. Level dependence of distortion-product otoacoustic emissions measured at high frequencies in humans. J. Acoust. Soc. Am. 2005, 117, 2980-2988. [CrossRef]

32. Robles, L.; Ruggero, M.A. Mechanics of the mammalian cochlea. Physiol. Rev. 2001, 81, 1305-1352. [CrossRef]

33. Katbamna, B.; Homnick, D.N.; Marks, J.H. Effects of chronic tobramycin treatment on distortion product otoacoustic emissions. Ear Hear. 1999, 20, 393-402. [CrossRef]

34. Abdala, C.; Dhar, S. Maturation and Aging of the Human Cochlea: A View through the DPOAE Looking Glass. J. Assoc. Res. Otolaryngol. 2012, 13, 403-421. [CrossRef]

35. Engdahl, B.; Kemp, D.T. The effect of noise exposure on the details of distortion product otoacoustic emissions in humans. J. Acoust. Soc. Am. 1996, 99, 1573-1587. [CrossRef] 
36. Namyslowski, G.; Morawski, K.; Trybalska, G.; Urbaniec, P. The latencies of the 2f1-f2 DPOAE measured using phase gradient method in young adults and in workers chronically exposed to noise. Otolaryngol. Pol. 2004, 58, 131-138.

37. Telischi, F.F.; Stagner, B.B.; Widick, M.P.; Balkany, T.J.; Lonsbury-Martin, B.L. Distortion-product otoacoustic emission monitoring of cochlear blood flow. Laryngoscope 1998, 108, 837-842. [CrossRef]

38. Neely, S.T.; Liu, Z. EMAV: Otoacoustic Emission Averager; Technical Memo No. 17; Boys Town National Research Hospital: Omaha, NE, USA, 1993.

39. Poling, G.; Lee, J.; Siegel, J.; Dhar, S. Clinical Utilisation of High-frequency DPOAEs. ENT Audiol. News 2012, 21, 1-6.

40. Heitmann, J.; Waldmann, B.; Schnitzler, H.U.; Plinkert, P.K.; Zenner, H.P. Suppression of distortion product otoacoustic emissions (DPOAE) near 2f1-f2 removes DP-gram fine structure-Evidence for a secondary generator. J. Acoust. Soc. Am. 1998, 103, 1527-1531. [CrossRef]

41. Talmadge, C.L.; Long, G.R.; Tubis, A.; Dhar, S. Experimental confirmation of the two-source interference model for the fine structure of distortion product otoacoustic emissions. J. Acoust. Soc. Am. 1999, 105, 275-292. [CrossRef]

42. Dhar, S.; Talmadge, C.L.; Long, G.R.; Tubis, A. Multiple internal reflections in the cochlea and their effect on DPOAE fine structure. J. Acoust. Soc. Am. 2002, 112, 2882-2897. [CrossRef]

43. Conrad, A.; Dreisbach, L. Repeatability of high-frequency DPOAE measures in normal-hearing children. Am. Audit. Soc. Abstr. 2011, 36, 42.

44. Newman, S.; Dreisbach, L. Repeatability of high-frequency behavioral and DPOAE measures in normal-hearing children. Am. Audit. Soc. Abstr. 2012, 37, 57.

45. Franklin, D.J.; McCoy, M.J.; Martin, G.K.; Lonsbury-Martin, B.L. Test/retest reliability of distortion-product and transiently evoked otoacoustic emissions. Ear Hear. 1992, 13, 417-429. [CrossRef]

46. Roede, J.; Harris, F.P.; Probst, R.; Xu, L. Repeatability of distortion product otoacoustic emissions in normally hearing humans. Audiology 1993, 32, 273-281. [CrossRef]

47. Beattie, R.C.; Kenworthy, O.T.; Luna, C.A. Immediate and short-term reliability of distortion-product otoacoustic emissions. Int. J. Audiol. 2003, 42, 348-354. [CrossRef]

48. Reavis, K.M.; McMillan, G.; Austin, D.; Gallun, F.; Fausti, S.A.; Gordon, J.S.; Helt, W.J.; Konrad-Martin, D. Distortion-product otoacoustic emission test performance for ototoxicity monitoring. Ear Hear. 2011, 32, 61-74. [CrossRef]

49. Dille, M.; McMillan, G.; Reavis, K.; Jacobs, P.; Fausti, S.; Konrad-Martin, D. Ototoxicity risk assessment combining distortion product otoacoustic emissions with a cisplatin dose model. J. Acoust. Soc. Am. 2010, 128, 1163-1174. [CrossRef]

50. Shera, C.A.; Guinan, J.J. Evoked otoacoustic emissions arise by two fundamentally different mechanisms: A taxonomy for mammalian OAEs. J. Acoust. Soc. Am. 1999, 105, 782-798. [CrossRef]

51. Martin, G.K.; Stagner, B.B.; Jassir, D.; Telischi, F.F.; Lonsbury-Martin, B.L. Suppression and enhancement of distortion-product otoacoustic emissions by interference tones above $\mathrm{f}(2)$. I. Basic findings in rabbits. Hear. Res. 1999, 136, 105-123. [CrossRef]

52. Rao, A.; Long, G.R. Effects of aspirin on distortion product fine structure: Interpreted by the two-source model for distortion product otoacoustic emissions generation. J. Acoust. Soc. Am. 2011, 129, 792-800. [CrossRef]

(C) 2019 by the authors. Licensee MDPI, Basel, Switzerland. This article is an open access article distributed under the terms and conditions of the Creative Commons Attribution (CC BY) license (http://creativecommons.org/licenses/by/4.0/). 